\title{
Mathematical modelling of the overflowing cylinder experiment
}

\author{
By P. D. HOWELL $L^{1} \dagger$ AND C. J. W. BREWARD \\ ${ }^{1}$ School of Mathematical Sciences, University of Nottingham, University Park, \\ Nottingham NG7 2RD \\ ${ }^{2}$ Mathematical Institute, University of Oxford, 24-29 St Giles', Oxford OX1 3LB
}

(Received 27 August 2004)

The overflowing cylinder (OFC) is an experimental apparatus designed to generate a controlled straining flow at a free surface, whose dynamic properties may then be investigated. Surfactant solution is pumped up slowly through a vertical cylinder. On reaching the top, the liquid forms a flat free surface which expands radially before overflowing down the side of the cylinder. The velocity, surface tension and surfactant concentration on the expanding free surface are measured using a variety of non-invasive techniques.

A mathematical model for the OFC has been previously derived by Breward, Darton, Howell \& Ockendon (2001) and shown to give satisfactory agreement with experimental results. However, a puzzling indeterminacy in the model renders it unable to predict one scalar parameter (e.g. the surfactant concentration at the centre of the cylinder), which must be therefore be taken from the experiments.

In this paper we analyse the OFC model asymptotically and numerically. We show that solutions typically develop one of two possible singularities. In the first, the surface concentration of surfactant reaches zero a finite distance from the cylinder axis, while $\dagger$ Present address: Mathematical Institute, University of Oxford, 24-29 St Giles', Oxford OX1 3LB 
the surface velocity tends to infinity there. In the second, the surfactant concentration is exponentially large and a stagnation point forms just inside the rim of the cylinder. We propose a criterion for selecting the free parameter, based on the elimination of both singularities, and show that it leads to good agreement with experimental results.

\section{Introduction}

\subsection{Background}

A surfactant is a chemical in solution in a liquid that (i) adsorbs preferentially at interfaces between the liquid and the surrounding atmosphere and (ii) when there reduces the local surface energy (Atkins 1992). A limiting case is that of insoluble surfactant which resides only at the interfaces. Many flows of practical importance to industry and medicine are influenced, beneficially or otherwise, by the presence of surfactant. Moreover, the special properties of surfactants are increasingly being exploited in novel technologies and therapies.

This paper concerns an apparatus called the overflowing cylinder (OFC), which is designed to investigate these properties under controlled conditions. An outline of the setup and the principal experimental observations are given below in $\S 1.2$; more details may be found in Bain, Manning-Benson \& Darton (2000); Manning-Benson (1998); Manning-Benson, Bain \& Darton (1997a); Manning-Benson, Bain, Darton, Sharpe, Eastoe \& Reynolds (1997b); Manning-Benson, Parker, Bain \& Penfold (1998).

A theoretical understanding of the fluid mechanics of the OFC is required so that the experimental results may be properly understood and applied to practical surfactant flows. To this end, a simple mathematical model has been developed in Breward, Darton, Howell \& Ockendon (2001). The agreement between the theory and experiments was 
found to be encouraging, although a full comparison has thus far been impossible, since one scalar quantity remains mathematically indeterminate. In other words, the model admits a one-parameter family of solutions because of a degeneracy in the equations describing the propagation of surfactant in an interface. In this paper we present numerical and asymptotic solutions of the model given in Breward et al. (2001). As well as offering a possible selection mechanism to resolve the indeterminacy, and allowing experimental results to be more thoroughly analysed, our solutions shed further light on surfactant flows in general, and on their propensity to develop spontaneous singularities in particular.

Of the previous models that have been proposed for flows involving surfactant, many describe the transport of insoluble surfactant by convection and surface diffusion over either a thin film (Halpern \& Grotberg 1992; Jensen 1998; Jensen \& Halpern 1998) or a deep layer of liquid (Di Pietro, Huh \& Cox 1978; Di Pietro \& Cox 1980; Jensen 1995). In either case, the deformation of the liquid-air interface under the surfactant-driven Marangoni flow may have a significant influence. When considering the behaviour of soluble surfactant in a thin liquid film, it is common to use the well-mixed approximation, in which convection in the bulk is negligible so that the bulk concentration profile is uniform or linear (see Jensen \& Grotberg 1993; Breward \& Howell 2002). In our model, however, the surfactant is soluble and bulk convection plays a major role in its transport, as in Harper \& Dixon (1974) and Harper (1992). On the other hand, gravity dominates the behaviour of the free surface, so that deformation of the interface may be neglected.

Our model equations for the liquid velocity and surfactant transport in the OFC are presented and in $\S 2$ and solved numerically in $\S 3$. The singularities observed in our numerical solutions are analysed asymptotically in $\S 4$. Finally, our results are compared with experiments and the conclusions are drawn in $§ 5$. 


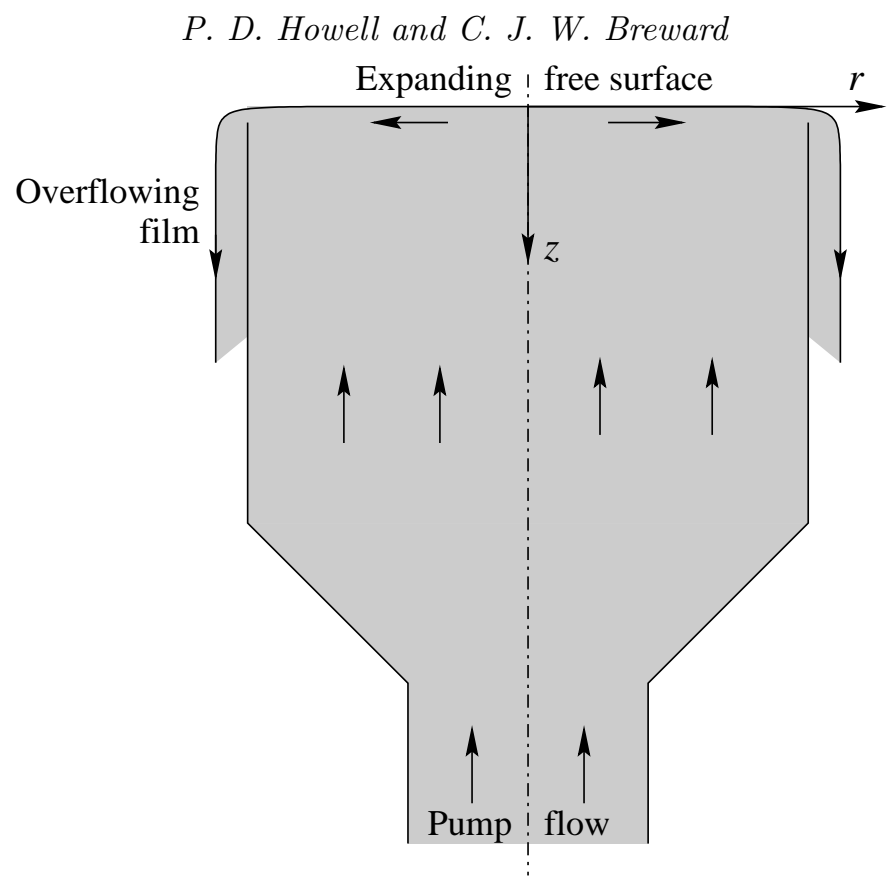

FIGURE 1. Schematic of the overflowing cylinder apparatus.

\subsection{Description of the experiment}

The OFC is shown schematically in figure 1. Surfactant solution is pumped slowly up through a circular cylinder with radius $4 \mathrm{~cm}$ (other sizes between 2.5 and $4 \mathrm{~cm}$ have also been used). Flow straighteners ensure an approximately uniform vertical flow of a few millimetres per second at the base of the cylinder. On reaching the top, the liquid forms a flat free surface which expands radially before overflowing down the side of the cylinder. A variety of independent, noninvasive techniques are used to measure the liquid velocity, surfactant concentration and surface tension in a neighbourhood of the cylinder axis. The main experimental observations to be explained are the following.

(a) The surface velocity $U_{s}$ is zero at the axis and increases approximately linearly with distance $r$ from the stagnation point. The strength $\mathrm{d} U_{s} / \mathrm{d} r$ of this straining flow depends strongly on the concentration of surfactant in the solution, varying from around $0.1 \mathrm{~s}^{-1}$ for pure water to $2.5 \mathrm{~s}^{-1}$ for a 0.6 -molar solution. 
(b) The surface concentration $\Gamma$ takes a well-defined finite value $\Gamma(0)$ at the axis, and has a slight quadratic decrease with $r$. The value $\Gamma(0)$ is quite different from that which would be found with the same bulk concentration under static conditions.

(c) The results are found to be virtually independent of the pump velocity, so long as it is large enough to allow the liquid to overflow steadily.

(d) The cylinder radius likewise appears to have negligible effect on the measurements taken near the axis. Furthermore, the details of the flow over the edge, which has been modified using various flanges, seem to be unimportant.

The OFC provides an expanding free surface whose parameters may readily be controlled and whose properties are relatively easy to measure. As indicated by the observations above, these properties may differ significantly from those of a static interface, and the influence of the surfactant is expected to be particularly dramatic for an expanding interface. Indeed, in practical applications, the ability of surfactant to assist or oppose surface expansion is one of its most significant features.

\section{Mathematical model}

A detailed derivation of our model for the OFC may be found in Breward et al. (2001). Here we simply outline the main assumptions made and present the resulting equations. The flow is assumed to be steady and radially symmetric, so the independent variables are the cylindrical polar coordinates $r$ and $z$, measuring distance from the cylinder axis and vertical depth respectively, as indicated in figure 1. The dependent variables are the water velocity $\boldsymbol{u}$, with components $u$ and $w$ in the $r$ - and $z$-directions, the bulk concentration $C$ of surfactant in solution and the surface concentration $\Gamma$ of surfactant adsorbed at the free surface. Gravity is assumed to be strong enough (i.e. the Froude number is assumed to be small enough) to keep the free surface effectively flat, and the 
origin of coordinates is chosen to locate it at $z=0$. The edge of the cylinder is given by $r=a$, where $a$ is the radius of the cylinder.

The solution is fed into the cylinder at a uniform bulk concentration $C_{b}$ with a pump flux $Q_{p}$. The governing equations, presented below in dimensionless form, are essentially the axially-symmetric boundary layer equations for $\boldsymbol{u}$ (with density $\rho$ and shear viscosity $\mu$ ) and a convection-diffusion equation for $C$ (with diffusion coefficient $D$ ). At the free surface $z=0$, boundary conditions are imposed representing conservation of surfactant and a balance between tangential viscous stress and surface-tension gradient. To close the problem, we require two constitutive equations relating the bulk and surface concentrations and the surface tension $\sigma$. The interface is assumed to be at thermodynamic equilibrium, which implies that functional relationships exist between $\sigma$ and $\Gamma$, and between $\Gamma$ and the sub-surface concentration $C_{s}(r)=C(r, 0)$. We employ the widely-used Langmuir isotherm and Frumkin equation (Adamson 1982), given respectively by

$$
\Gamma=\frac{\Gamma_{\mathrm{sat}} C_{s}}{k+C_{s}}, \quad \sigma-\sigma_{w}=R T \Gamma_{\mathrm{sat}} \log \left(1-\frac{\Gamma}{\Gamma_{\mathrm{sat}}}\right) .
$$

Here, $\sigma_{w}$ is the surface tension of pure water, $R$ is the gas constant and $T$ is the absolute temperature. The parameters $\Gamma_{\text {sat }}$ and $k$, representing the maximum possible surface concentration and the bulk concentration at which $\Gamma=\Gamma_{\text {sat }} / 2$ respectively, are determined from static experiments.

We nondimensionalise the variables as follows:

$$
C=C_{b} \tilde{C}, \quad z=\frac{\Gamma_{\mathrm{sat}}}{C_{b}} \tilde{z}_{d}=\frac{\Gamma_{\mathrm{sat}}}{\delta C_{b}} \tilde{z}_{h}, \quad w=\frac{D C_{b}}{\delta \Gamma_{\mathrm{sat}}} \tilde{w}, \quad r=L \tilde{r}, \quad u=\frac{D C_{b}^{2} L}{\Gamma_{\mathrm{sat}}^{2}} \tilde{u},
$$

where

$$
\delta=\sqrt{\frac{\rho D}{\mu}}, \quad L=\frac{\Gamma_{\mathrm{sat}}^{2} \sqrt{R T}}{C_{b}^{3 / 2}\left(\rho \mu D^{3}\right)^{1 / 4}} .
$$

The distinguished lengthscale $L$ represents the distance over which we expect $\Gamma$ to vary significantly. Notice that the dimensional thicknesses of the diffusive and hydrodynamic 
boundary layers differ by a factor of $\delta$ (the inverse square root of the Prandtl number), which is small for all aqueous surfactant solutions of interest. For this reason, two different dimensionless variables $\tilde{z}_{d}$ and $\tilde{z}_{h}$ are used for the bulk concentration and velocity fields respectively, i.e. $\bar{C}=\bar{C}\left(\bar{x}, \bar{z}_{d}\right), \overline{\boldsymbol{u}}=\overline{\boldsymbol{u}}\left(\bar{x}, \bar{z}_{h}\right)$. For ease of notation we henceforth drop these subscripts and the tildes.

The dimensionless boundary-layer equations and boundary conditions read

$$
\begin{aligned}
u \frac{\partial u}{\partial r}+w \frac{\partial u}{\partial z} & =\frac{\partial^{2} u}{\partial z^{2}}+\frac{\epsilon^{2}}{r_{0}} F\left(\frac{r}{r_{0}}\right) F^{\prime}\left(\frac{r}{r_{0}}\right) \\
\frac{1}{r} \frac{\partial}{\partial r}(r u)+\frac{\partial w}{\partial z} & =0 \\
u & =U_{s}(r) \text { on } z=0 \\
w & =0 \text { on } z=0 \\
u \rightarrow \epsilon F\left(\frac{r}{r_{0}}\right) \text { as } z \rightarrow \infty & \\
u=0 \text { on } r & =0 .
\end{aligned}
$$

The function $F\left(r / r_{0}\right)$ represents the bulk velocity at infinity and is found, by solving for an inviscid flow that is uniform as $z \rightarrow \infty$ and has a line sink around the rim, to be

$$
F(\rho)=\frac{1}{\pi} \int_{0}^{\infty} \frac{\mathrm{I}_{1}(k \rho)}{\mathrm{I}_{1}(k)} \mathrm{d} k .
$$

As shown in figure 2, $F(\rho)$ tends to infinity as $\rho \rightarrow 1$. This is a consequence of our assumption that gravity dominates, forcing the free surface to be flat. In this limit, the edge of the cylinder becomes a line sink, which removes fluid at the same rate at which it is introduced by the pump. In practice, there must be a region near $r=a$ where our zero-Froude-number limit breaks down, and the liquid flows over the edge of the cylinder at a nonzero (but small) height with a finite (but large) velocity. We do not attempt to model this complicated weir flow here. 


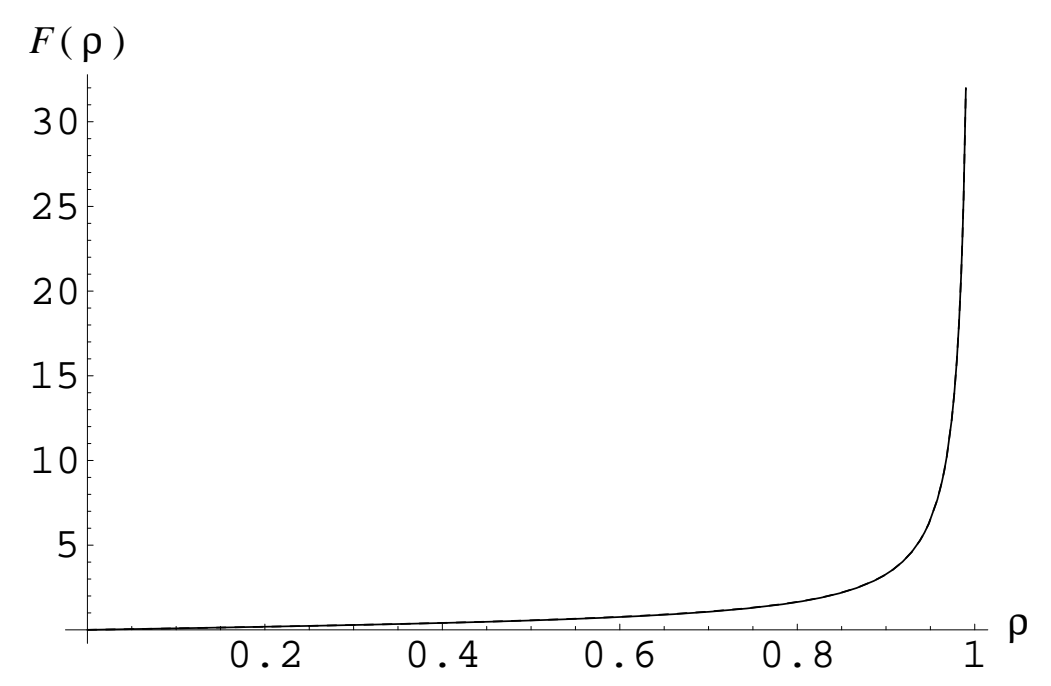

Figure 2. The function $F(\rho)$ given by (2.10).

The corresponding equations for $C$ are

$$
\begin{gathered}
U_{s}(r) \frac{\partial C}{\partial r}-\frac{1}{r} \frac{\mathrm{d}}{\mathrm{d} r}\left(r U_{s}\right) z \frac{\partial C}{\partial z}=\frac{\partial^{2} C}{\partial z^{2}}, \\
C=C_{s}(r) \text { on } z=0, \\
C \rightarrow 1 \text { as } z \rightarrow \infty, \\
\frac{\partial C}{\partial r}=0 \text { on } r=0 .
\end{gathered}
$$

The problems for $\boldsymbol{u}$ and $C$ are coupled via the two remaining boundary conditions, representing conservation of adsorbed surfactant and a balance of surface tangential stress:

$$
\begin{aligned}
\frac{\partial C}{\partial z}=\frac{1}{r} \frac{\mathrm{d}}{\mathrm{d} r}\left(\frac{r U_{s} C_{s}}{\beta+C_{s}}\right) \text { on } z & =0, \\
\frac{\partial u}{\partial z} & =\frac{1}{\beta+C_{s}} \frac{\mathrm{d} C_{s}}{\mathrm{~d} r} \text { on } z=0 .
\end{aligned}
$$

Equations (2.4-2.16) constitute our dimensionless model for $u, w, C, U_{s}$ and $C_{s}$. Both $\Gamma$ and $\sigma$ have been eliminated from $(2.15,2.16)$ in favour of $C_{s}$ by using $(2.1)$. 


\begin{tabular}{lclc} 
Quantity & Symbol Units & Approx. value \\
\hline Cylinder radius & $a$ & $\mathrm{~m}$ & $4 \times 10^{-2}$ \\
Pump flux & $Q_{p}$ & $\mathrm{~m}^{3} \mathrm{~s}^{-1}$ & $1.6 \times 10^{-5}$ \\
Liquid density & $\rho$ & $\mathrm{kg} \mathrm{m}^{-3}$ & $10^{3}$ \\
Liquid viscosity & $\mu$ & $\mathrm{kg} \mathrm{m}^{-1} \mathrm{~s}^{-1}$ & $10^{-3}$ \\
Bulk concentration & $C_{b}$ & $\mathrm{~mol} \mathrm{~m}^{-3}$ & 0.58 \\
Langmuir constant & $k$ & $\mathrm{~mol} \mathrm{~m}^{-3}$ & 0.08 \\
Langmuir constant & $\Gamma_{\mathrm{sat}}$ & $\mathrm{mol} \mathrm{m}^{-2}$ & $4 \times 10^{-6}$ \\
Diffusion coefficient & $D$ & $\mathrm{~m}^{2} \mathrm{~s}^{-1}$ & $5 \times 10^{-10}$ \\
Surface energy & $R T$ & $\mathrm{~J} \mathrm{~mol}^{-1}$ & $2.5 \times 10^{3}$ \\
\hline
\end{tabular}

TABLE 1. Typical dimensional parameter values for the overflowing cylinder.

The three dimensionless groups remaining in the problem are

$$
r_{0}=\frac{a}{L} \approx 2, \quad \beta=\frac{k}{C_{b}} \approx 0.16, \quad \epsilon=\frac{Q_{p} \Gamma_{\mathrm{sat}}^{2}}{\pi a^{3} D C_{b}^{2}} \approx 0.01
$$

which have been estimated using the typical dimensional parameter values for the OFC given in table 1 . The fact that $r_{0}$ is order one implies that the length-scale over which the surface concentration varies significantly is the same order as the cylinder radius. Although it appears to be fairly small, it is safest to treat $\beta$ as an order one constant also, since the limit $\beta \rightarrow 0$ fails at low values of $C_{s}$. Finally, the smallness of $\epsilon$ reflects the fact that the pump velocity is much less than the surfactant-induced surface velocity, and explains the lack of dependence of the experimental results on $Q_{p}$. Notice, though, that $\epsilon$ multiplies the function $F\left(r / r_{0}\right)$ which tends to infinity as $r \rightarrow r_{0}$, so the limit $\epsilon \rightarrow 0$ is nonuniform in a neighbourhood of $r=r_{0}$.

As shown in Breward et al. (2001), the equations and boundary conditions presented 
above do not determine a unique solution near $r=0$. One further piece of information is required, and we choose to specify the concentration at the axis of the cylinder:

$$
C_{s}(0)=C_{0}
$$

If $C_{0}$ is assumed to be known, then the local behaviour of $\boldsymbol{u}$ and $C$ as $r \rightarrow 0$ may be found from (2.4-2.16). In particular, the strength of the stagnation-point flow is given by

$$
U_{s}^{\prime}(0)=\frac{1}{\pi}\left(\frac{\left(\beta+C_{0}\right)\left(1-C_{0}\right)}{C_{0}}\right)^{2} .
$$

However, the concentration at the origin is an important experimentally-measured quantity that ought to be a prediction of the model rather than a parameter to be specified.

To elucidate this indeterminacy, Breward et al. (2001) constructed an ad hoc paradigm equation, based on conservation of surfactant coupled with a simple rule for the acceleration of the surface velocity by a gradient in surface concentration. Their model equation resembles the porous medium equation and has the following properties. As for the full model (2.4-2.16), the concentration $C_{0}$ at the origin must be specified before a unique solution may be found. If a relatively small value of $C_{0}$ is chosen, then the concentration reaches zero at a finite value of $r<r_{0}$. For larger values of $C_{0}$, however, the concentration blows up as $r \rightarrow r_{0}$. This led Breward et al. (2001) to suggest that a unique value of $C_{0}$ might be selected by requiring the concentration to be nonzero in $r<r_{0}$ and finite as $r \rightarrow r_{0}$. In the following section we present numerical solutions of (2.4-2.16) and show that this behaviour of the paradigm equation from Breward et al. (2001) is shared by the full model. 


\section{Numerical solution}

\subsection{Discretisation}

We use a simple scheme that is second-order in $z$ and treats the diffusive terms implicitly. A first-order difference is used to march in the $r$-direction, such that the problem to be solved at each new value of $r$ is linear. We truncate at a finite depth $L$ in the $z$-direction and discretise the interval $[0, L]$ using a uniform mesh: $z_{i}=i \Delta z, \Delta z=L /(N+1)$ for some integer $N$. At each value of $r$, the approximate solution is denoted by

$$
u_{i}=u\left(r, z_{i}\right), \quad w_{i}=w\left(r, z_{i}\right), \quad C_{i}=C\left(r, z_{i}\right)
$$

while that obtained at the previous $r$-step is given by

$$
\bar{u}_{i}=u\left(r-\Delta r, z_{i}\right), \quad \bar{w}_{i}=w\left(r-\Delta r, z_{i}\right), \quad \bar{C}_{i}=C\left(r-\Delta r, z_{i}\right) .
$$

Instead of $w_{i}$, it is convenient to obtain the solution in terms of

$$
W_{i}=\frac{\Delta r}{\Delta z} w_{i}, \quad \bar{W}_{i}=\frac{\Delta r}{\Delta z} \bar{w}_{i}
$$

The boundary-layer equation (2.4) is approximated by

$$
\bar{u}_{i}\left(u_{i}-\bar{u}_{i}\right)+\frac{W_{i}}{2}\left(\bar{u}_{i+1}-\bar{u}_{i-1}\right)=\epsilon^{2} \bar{F}(F-\bar{F})+\frac{\Delta r}{\Delta z^{2}}\left(u_{i+1}-2 u_{i}+u_{i-1}\right),
$$

where $W_{i}$ is found from $(2.5,2.7)$ using (e.g.) the trapezium rule,

$$
\begin{aligned}
W_{0} & =0 \\
W_{i+1} & =W_{i}-\frac{1}{2}\left\{u_{i+1}+u_{i}-\left(1-\frac{\Delta r}{r}\right)\left(\bar{u}_{i+1}+\bar{u}_{i}\right)\right\} .
\end{aligned}
$$

To avoid having to evaluate the integral (2.10) for the subsurface velocity $F\left(r / r_{0}\right)$ repeatedly, we use an approximation for $F$, given in Appendix A.

The advection-diffusion equation (2.11) for $C$ is discretised via

$$
\bar{u}_{0}\left(C_{i}-\bar{C}_{i}\right)-\frac{i}{2}\left(\bar{C}_{i+1}-\bar{C}_{i-1}\right)\left[u_{0}-\left(1-\frac{\Delta r}{r}\right) \bar{u}_{0}\right]=\frac{\Delta r}{\Delta z^{2}}\left(C_{i+1}-2 C_{i}+C_{i-1}\right) .
$$


The matching conditions $(2.8,2.13)$ are applied at the truncated depth $L$,

$$
u_{n+1}=\epsilon F\left(r / r_{0}\right), \quad C_{n+1}=1,
$$

while the boundary conditions $(2.15,2.16)$ on $z=0$ give rise to

$$
\begin{aligned}
\frac{u_{1}-u_{-1}}{2 \Delta z} & =\frac{C_{0}-\bar{C}_{0}}{\Delta r\left(\beta+\bar{C}_{0}\right)}, \\
\frac{C_{1}-C_{-1}}{2 \Delta z} & =\frac{\bar{C}_{0}\left(u_{0}-\bar{u}_{0}\right)}{\Delta r\left(\beta+\bar{C}_{0}\right)}+\frac{\beta \bar{u}_{0}\left(C_{0}-\bar{C}_{0}\right)}{\Delta r\left(\beta+\bar{C}_{0}\right)^{2}}+\frac{\bar{u}_{0} \bar{C}_{0}}{r\left(\beta+\bar{C}_{0}\right)} .
\end{aligned}
$$

Now, at each new value of $r$, assuming that the solutions from the previous step (i.e. all variables with bars) are known, (3.4-3.9) gives a set of linear algebraic equations for $\left\{u_{i}, W_{i}, C_{i}\right\}$. The resulting sparse system is readily solved by elimination. The step $\Delta r$ in the $r$-direction is varied depending on the rate at which the solution changes between one step and the next. As pointed out in $\S 2$, to get the solution started, we have to choose a particular value of $C_{0}$.

\subsection{Results}

We evaluate $r_{0}, \beta$ and $\epsilon$ using the parameter values given in table 1 . The value of $C_{0}$ remains to be chosen (recall that $C_{0}$ is dimensionless, representing the ratio between the dimensional subsurface concentration at the origin and $C_{b}$ ). We start with a relatively low value of 0.3 and plot the resulting variation of $U_{s}$ and $C_{s}$ with $r$ in figure 3 . Near the origin, $U_{s}$ is linear as expected, and its slope agrees with the theoretical prediction (2.19). As suggested by Breward et al. (2001), a singularity, with $C_{s} \rightarrow 0$ and $U_{s} \rightarrow \infty$, develops at a finite value of $r \approx 1.62$, beyond which we are unable to continue the solution. The effects of refining the mesh and of increasing the truncation depth $L$ are indicated by the different curves in figure 3 ; since these are virtually indistinguishable, the scheme appears to have converged adequately.

The corresponding profiles of $u$ and $C$ versus depth are shown in figure 4 at various values of $r$. As the singularity is approached, we see that the variations in $u$ and $C$ are 

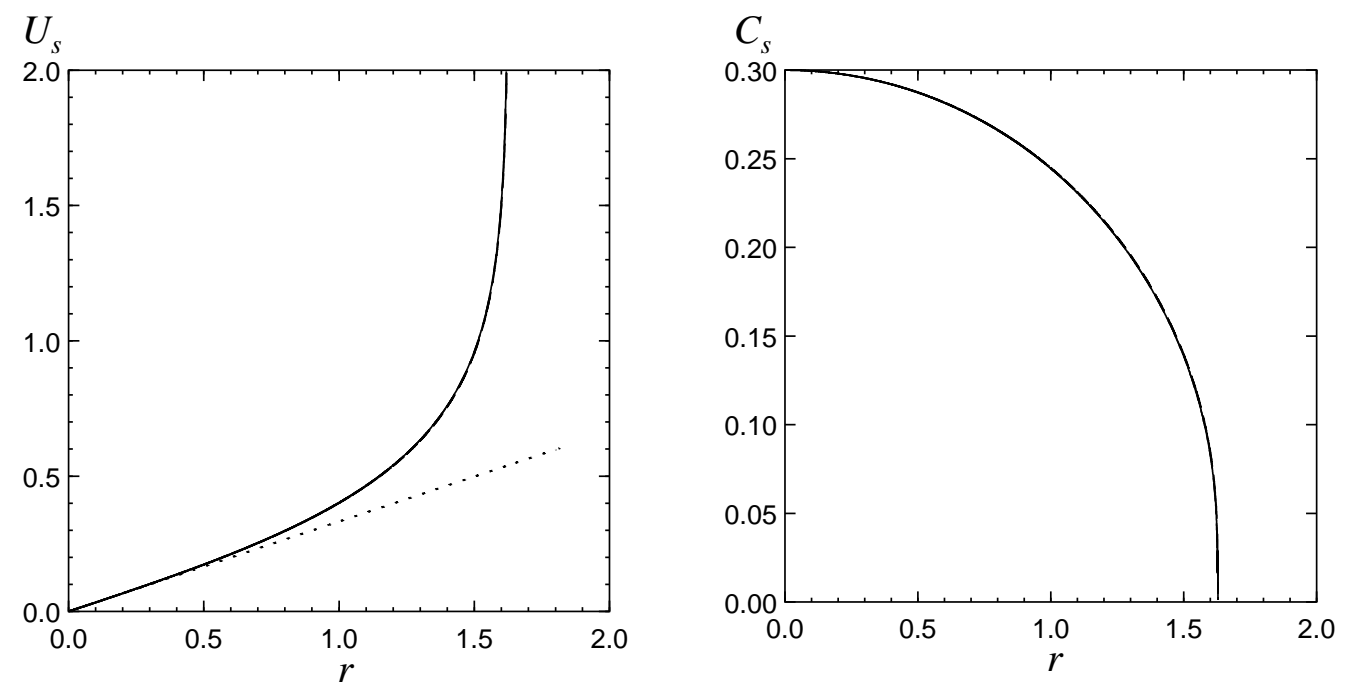

400 grid points; $L=6.0$

200 grid points; $L=6.0$

400 grid points; $L=10.0$

200 grid points; $L=10.0$

FIgURE 3. Surface velocity $U_{s}$ and subsurface concentration $C_{s}$ versus radial coordinate $r$, for the parameter values $C_{b}=0.58 \mathrm{~mol} \mathrm{~m}^{-3}, a=0.04 \mathrm{~m}, C_{0}=0.3$ so that $r_{0} \approx 2.332$. The four curves produced by varying the truncation depth and the number of grid points are indistinguishable. The dotted line is the asymptotic behaviour predicted by (2.19).
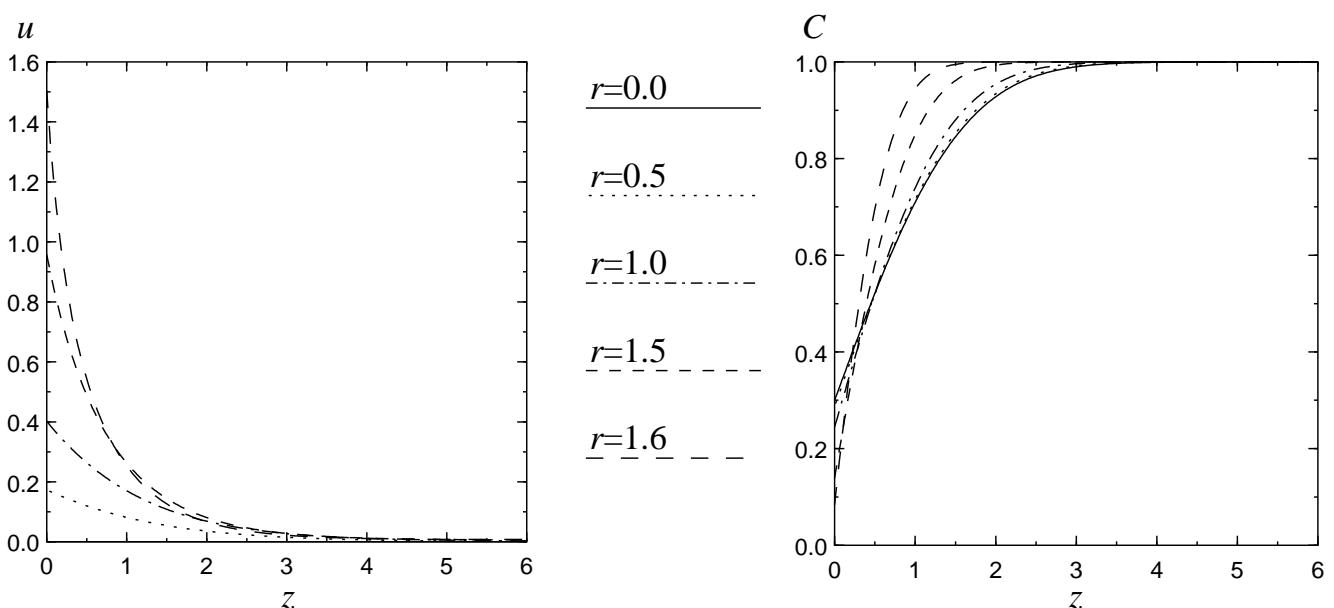

Figure 4 . Velocity $u$ and concentration $C$ versus depth $z$, for the same parameter values as in figure 3 . 

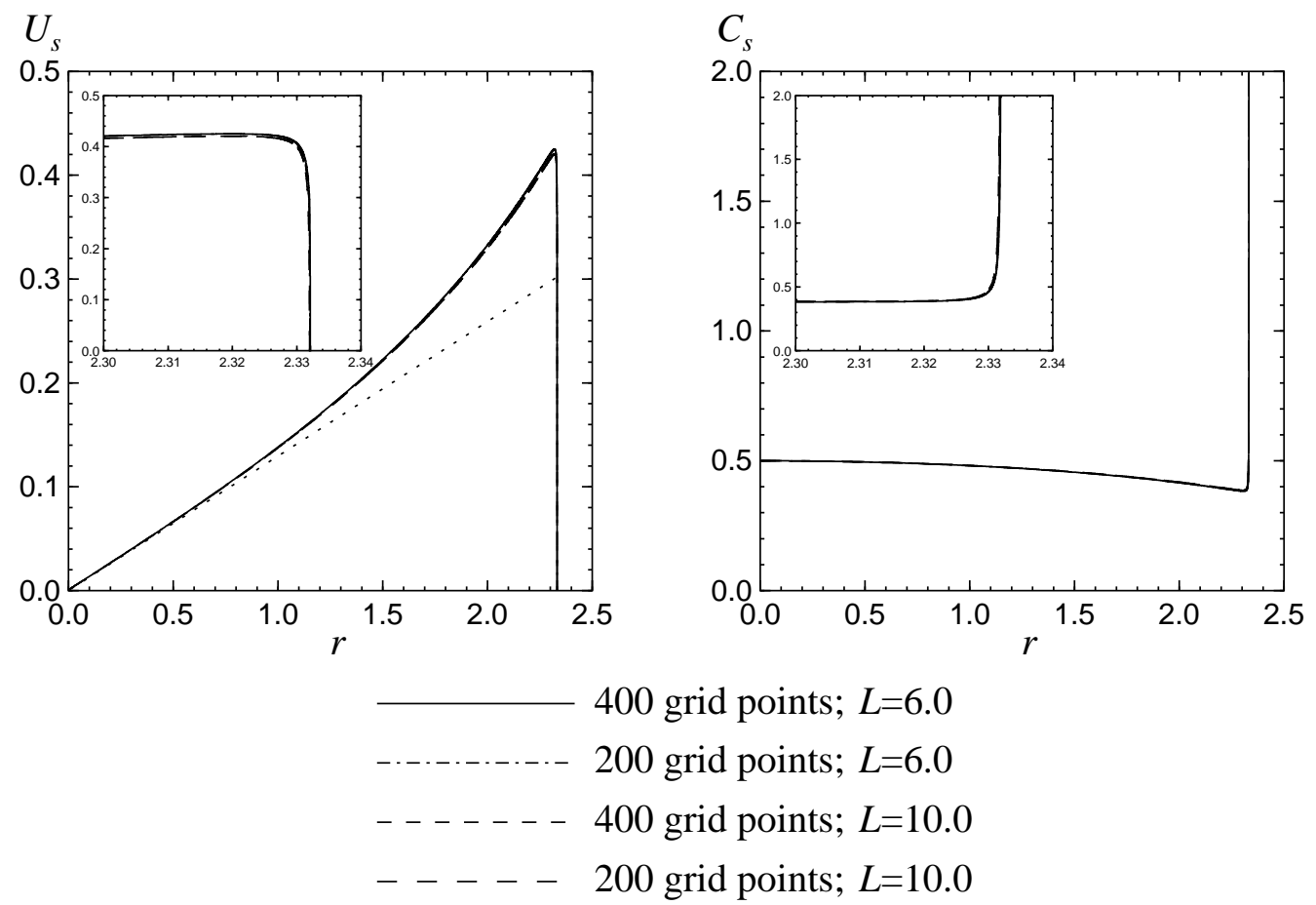

FiguRE 5. Surface velocity $U_{s}$ and subsurface concentration $C_{s}$ versus radial coordinate $r$, for the parameter values $C_{b}=0.58 \mathrm{~mol} \mathrm{~m}^{-3}, a=0.04 \mathrm{~m}, C_{0}=0.5$, so that $r_{0} \approx 2.332$. The dotted line is the asymptotic behaviour predicted by (2.19). The insets show the local behaviour near $r=r_{0}$.

confined to increasingly narrow boundary layers near $z=0$. Since the singularity occurs at a value of $r$ significantly less than $r_{0} \approx 2.332$ for these parameter values, the velocity at infinity remains very small throughout.

In figure 5 we show the behaviour of $U_{s}$ and $C_{s}$ for the same parameter values but a somewhat larger value of $C_{0}=0.5$. Again, the correct linear behaviour of $U_{s}$ is obtained near $r=0$, and the solutions are only very slightly influenced by variations in $N$ and $L$. Here, however, $C_{s}$ has not yet reached zero when $r$ approaches $r_{0} \approx 2.332$. Consequently, as predicted by Breward et al. (2001), $C_{s}$ tends to infinity as $r \rightarrow r_{0}$ while, at the same time, the surface velocity tends to zero. The close-ups of the behaviours of $U_{s}$ and $C_{s}$ near $r=r_{0}$ are given in the insets. 

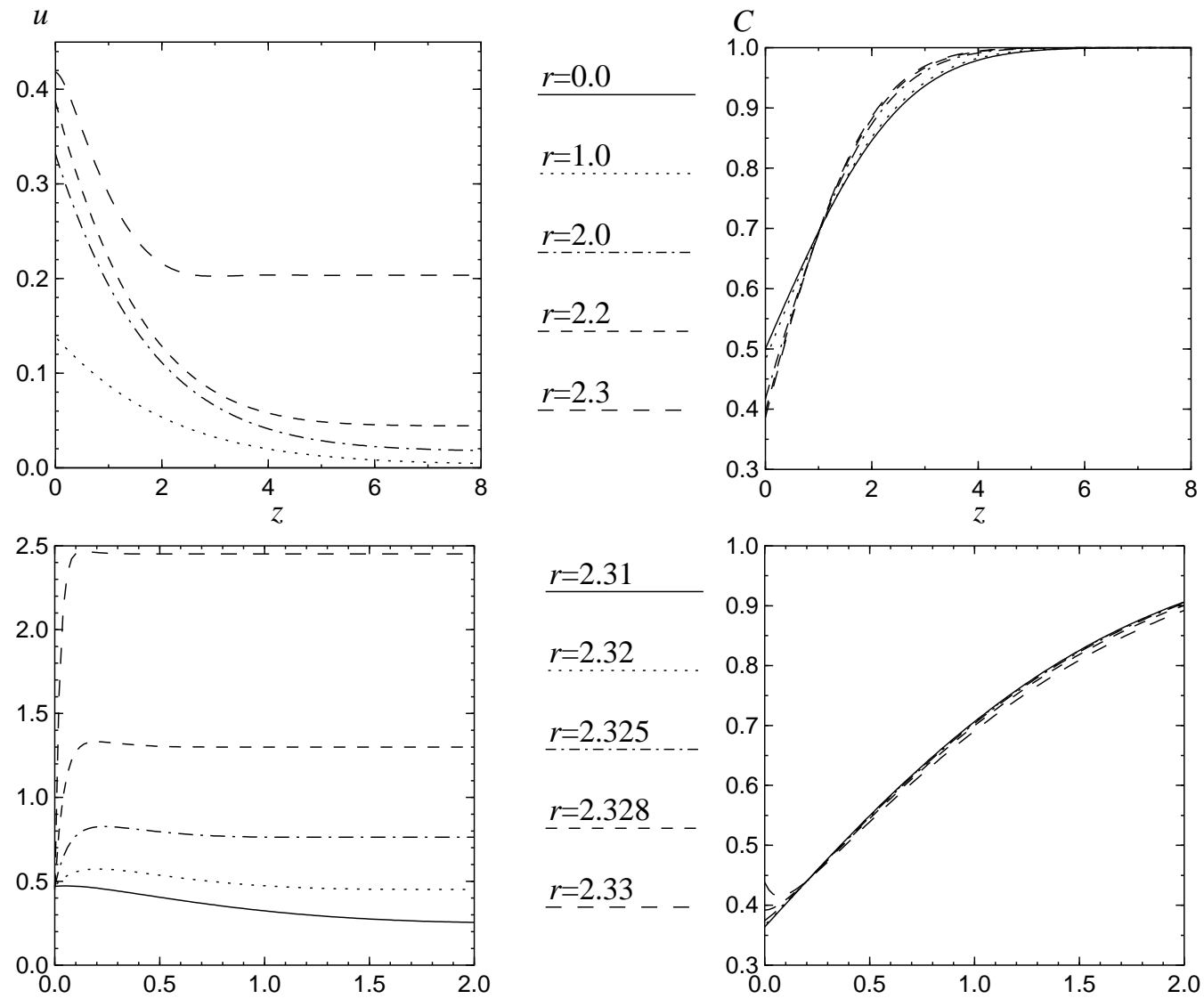

Figure 6 . Velocity $u$ and concentration $C$ versus depth $z$, for the same parameter values as in figure 5 .

The corresponding variations of $u$ and $C$ with $z$ are shown in figure 6 . Initially, the surface velocity increases while the velocity at depth remains small. As $r$ approaches $r_{0}$, the subsurface velocity catches up and finally overtakes the surface velocity, and we end up with a uniform plug flow that is brought to zero in a tiny boundary layer at the surface. Meanwhile, the concentration varies only slightly with $r$ until right at the end, when it starts to increase in a very narrow boundary layer near $z=0$.

By varying $C_{0}$ as a shooting parameter, we can attempt to obtain a distinguished solution that lies between these two generic cases, i.e. for which $C_{s}$ reaches zero exactly at $r=r_{0}$. The variation of $U_{s}$ and $C_{s}$ with $r$ are shown in figure 7 for a solution close 


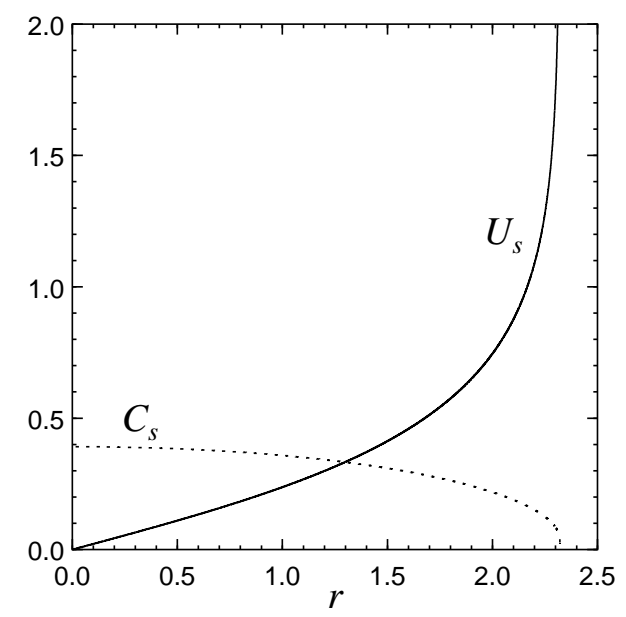

FiguRE 7. Surface velocity $U_{s}$ and subsurface concentration $C_{s}$ versus radial coordinate $r$, for the parameter values $C_{b}=0.58 \mathrm{~mol} \mathrm{~m}^{-3}, a=0.04 \mathrm{~m}, C_{0}=0.391$, so that $r_{0} \approx 2.332$.

to this critical one. It appears that the surface velocity again tends to infinity as the surface concentration tends to zero. In figure 8 we show the variations of $u$ and $C$ with depth in the same parameter regime for values of $r$ close to $r_{0}$. This special solution appears to have the property that the surface and subsurface velocities tend to infinity at approximately the same rate.

\section{Asymptotic behaviour near the singularities}

\subsection{Motivation}

Further support for the structure suggested by our numerical solutions may be obtained by examining the possible limiting forms of solutions to $(2.4-2.16)$. We begin in $\S 4.2$ by considering the behaviour as $C_{s} \rightarrow 0$, the aim being to confirm that solutions in which $C_{s}$ reaches zero at a finite value of $r<r_{0}$ really are a feature of the equations rather than a numerical artefact. Similarly, in $\S 4.3$ we examine the asymptotic behaviour near the edge $r=r_{0}$ of the cylinder, where the subsurface velocity tends to infinity. We show that generically $C_{s}$ grows exponentially while the surface velocity goes to zero, in agreement 
$u$
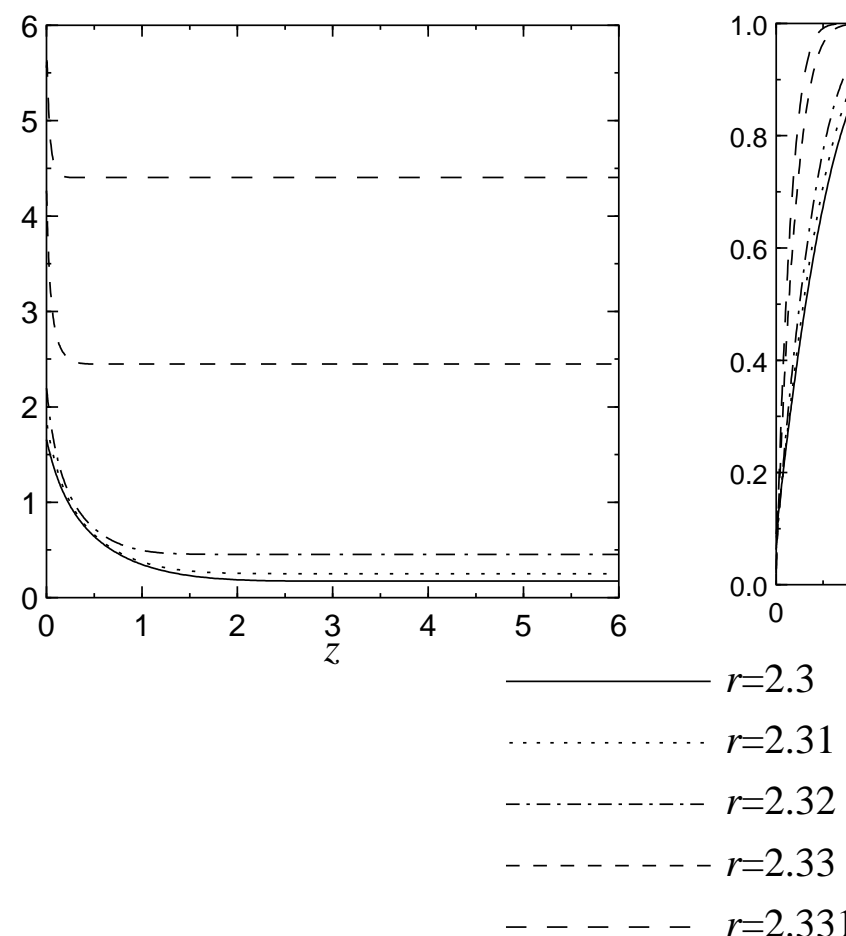

C

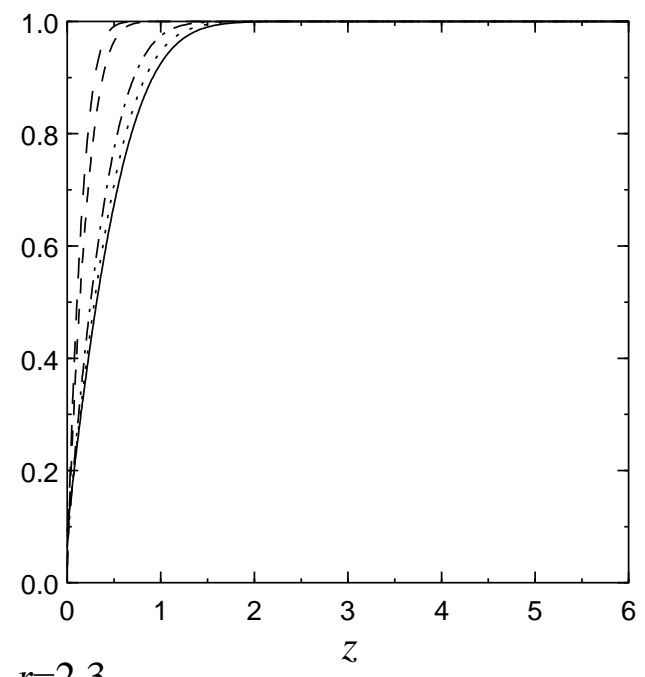

FigURE 8 . Velocity $u$ and concentration $C$ versus depth $z$, for the same parameter values as in figure 7 .

with our simulations. Finally, we show in $\S 4.4$ that there is a special solution in which $C_{s} \rightarrow 0$ as $r \rightarrow r_{0}$, while the surface and subsurface velocities approach infinity at roughly the same rate.

These analyses help to reassure us that our finite-difference scheme is producing sensible answers since, in each case, we show that there is good agreement between the asymptotic predictions and numerical results. Furthermore, they demonstrate local singular structures supported by the equations which, presumably, may arise in many other surfactant flows. 

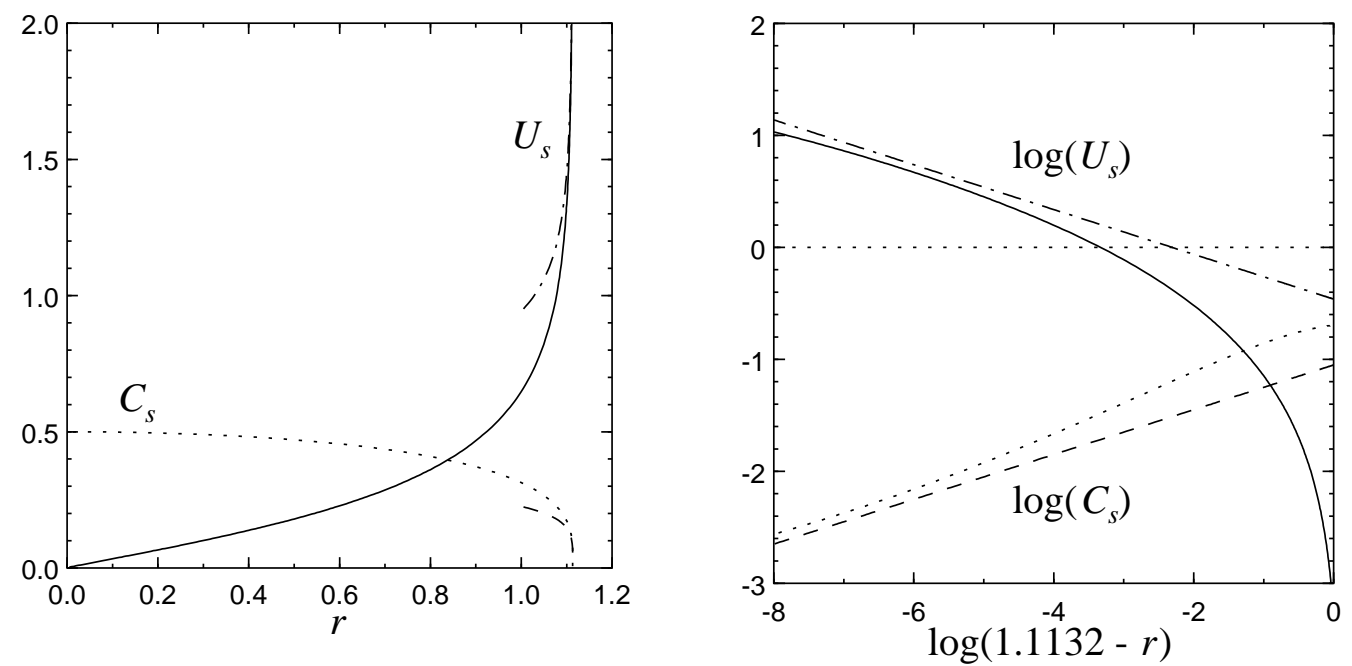

Figure 9. The graph on the left shows the variation of surface velocity $U_{s}$ (solid line) and surface concentration $C_{s}$ (dotted line) with $r$ for a solution with a finite-distance singularity. The right-hand graph is a corresponding $\log -\log$ plot. The dashed lines show the asymptotic behaviours $U_{s} \sim 0.63(1.1132-r)^{-1 / 5}, C_{s} \sim 0.35(1.1132-r)^{1 / 5}$. Parameter values $C_{b}=0.58$, $a=0.04, C_{0}=0.5 ; \beta$ is artificially increased to 0.5 to highlight the local behaviour.

\subsection{Behaviour as $C_{s} \rightarrow 0$}

We start by looking for solutions where $C_{s} \rightarrow 0$ and $U_{s} \rightarrow \infty$ as $r \rightarrow r^{*}$, for some value $r^{*}<r_{0}$, as shown in figure 3. The details of the appropriate rescaling and asymptotic behaviour of equations (2.4-2.16) may be found in Appendix B. We find that the solution takes the universal local form

$$
C_{s} \sim K_{1}\left(r^{*}-r\right)^{1 / 5}, \quad U_{s} \sim K_{2}\left(r^{*}-r\right)^{-1 / 5},
$$

where the constants $K_{1}$ and $K_{2}$ are related by

$$
K_{2}=\frac{\beta K_{1}^{3 / 2}}{A^{5 / 2}},
$$

and the numerical constant $A \approx 0.8868$.

The behaviour (4.1) only emerges when $C_{s}$ is much smaller than $\beta$, i.e. when $r^{*}-r \ll$ $\beta^{5}$. Since $\beta$ is typically rather small, it is difficult to obtain the exponents accurately 
using our simple numerical scheme. To highlight the local behaviour, we therefore use an artificially high value of $\beta=0.5$. Sample numerical plots of $U_{s}$ and $C_{s}$ are shown in figure 9, along with fitted functions of the form (4.1). A log-log plot indicates that the numerical solutions adopt the predicted power-law behaviour as the singularity is approached. Moreover, the fitted curves have $K_{1} \approx 0.63, K_{2} \approx 0.35$, which are close to satisfying (4.2), since $\beta K_{1}^{3 / 2} / A^{5 / 2} \approx 0.338$.

\subsection{Behaviour near the sink}

Now we consider the possible behaviour of solutions to (2.4-2.16) as $r$ approaches $r_{0}$, where the subsurface velocity $F\left(r / r_{0}\right)$ tends to infinity. Figure 5 indicates that the subsurface concentration approaches infinity while the surface velocity goes to zero in a very small neighbourhood of $r_{0}$. To explore this region further, we artificially increase the value of $\epsilon$ to 0.1 so that, as shown in figure 10, the range over which $C_{s}$ and $U_{s}$ vary is expanded.

The details of the local analysis are given in Appendix C. We find that the algebraic tending to infinity of the bulk velocity leads to an exponentially large subsurface concentration, with

$$
C_{s} \sim B \exp \left[\left(\frac{\epsilon r_{0}}{\pi}\right)^{3 / 2} \frac{2}{\sqrt{3}\left(r_{0}-r\right)}\right]
$$

for some constant $B$. Figure 10 shows that this predicted behaviour (with $B=0.46$ ) agrees with our simulations.

With the subsurface concentration given by (4.3), conservation of surfactant forces a gradient in the surface velocity, which culminates in the formation of a stagnation point a small but finite distance away from the sink at the edge of the cylinder. This does not appear at first glance to agree with the numerical results shown in figures 5 and 10. However, the unbounded growth of $C_{s}$, and consequent sending of $U_{s}$ to zero, 

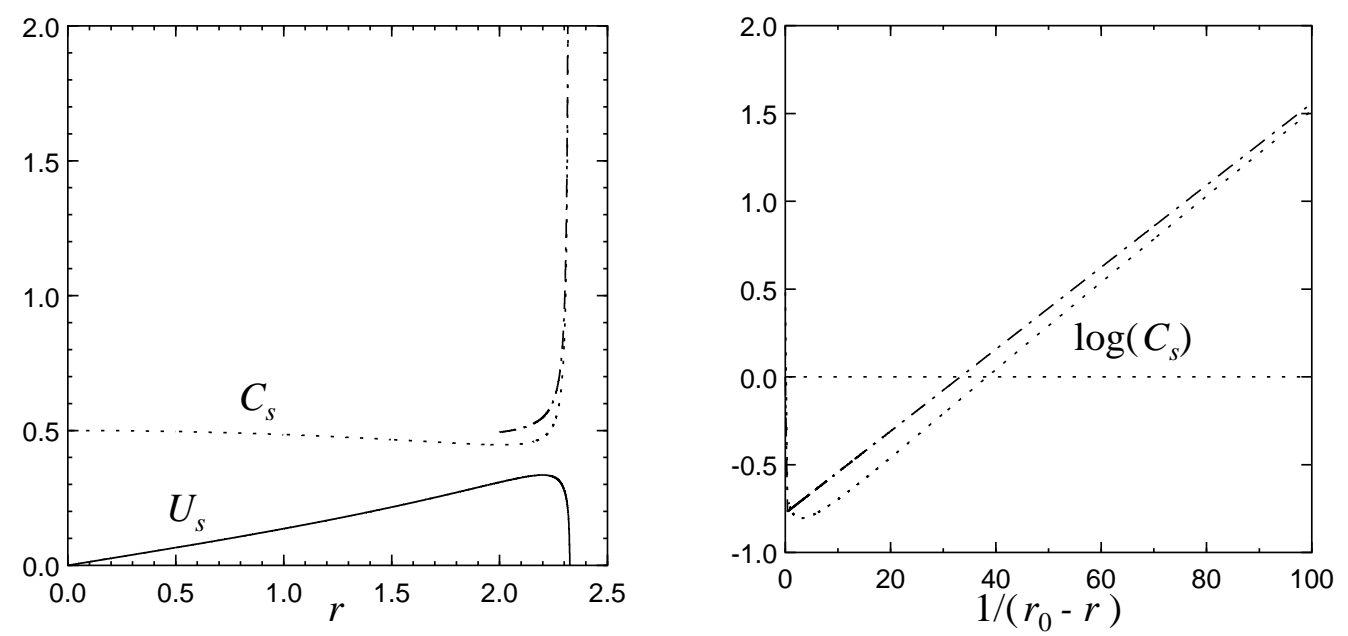

Figure 10. The left-hand graph shows the surface velocity $U_{s}$ and subsurface concentration $C_{s}$ versus radial coordinate $r$, for the parameter values $C_{b}=0.58 \mathrm{~mol} \mathrm{~m}^{-3}, a=0.04 \mathrm{~m}, C_{0}=0.5$; $\epsilon$ is artificially increased to 0.1 to highlight the local behaviour. The dot-dashed line shows the fit (C10) with $B=0.46$. The right-hand graph is the corresponding plot of $\log \left(C_{s}\right)$ versus $1 /\left(r_{0}-r\right)$.

only occurs in a layer near $r=r_{0}$ whose thickness is $O\left(\epsilon^{3 / 2}\right)$. For the parameter values used in figure $5, \epsilon \approx 0.007$, so that the point ( $\operatorname{say} r=r^{*}$ ) at which $U_{s}$ becomes zero is extremely close to $r=r_{0}$. With $\epsilon=0.1$, there is a noticeable difference, as can be seen in the close-up shown in figure 11.

The predicted local behaviour of $U_{s}(r)$ is

$$
U_{s} \sim \frac{\pi^{2}}{18 \gamma(3 / 4)^{4}}\left(\frac{\mathrm{d} C_{s}}{\mathrm{~d} r}\left(r^{*}\right)\right)^{2}\left(r^{*}-r\right)^{3}
$$

where $\gamma$ is the Gamma function. Close inspection of figure 11 reveals that $U_{s}$ does appear to go to zero smoothly, although our results do not agree particularly well with (4.4). For the parameter values used in figure 11, (4.4) takes the form

$$
U_{s} \approx 1.1 \times 10^{9}\left(r^{*}-r\right)^{3}
$$

and our numerical scheme is unable to reproduce this dramatic behaviour accurately.

What happens between the stagnation point and the sink is unclear, but it seems 


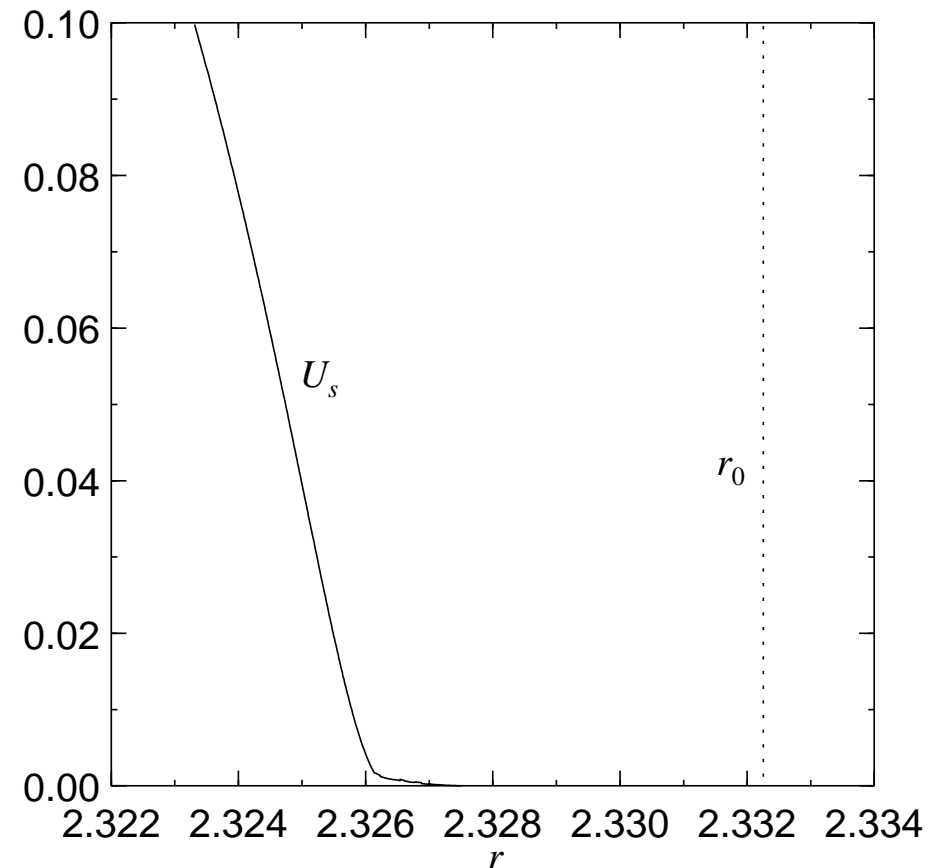

Figure 11. Close-up of the behaviour of $U_{s}$ from figure 10 near $r=r_{0}$.

highly unlikely that this situation could be stable, providing further support for our proposal to reject this kind of solution. In reality, as pointed out previously, the bulk velocity is bounded, although large, near the edge of the cylinder. Presumably the subsurface concentration starts to grow in a similar fashion to (4.3), but is cut off in a small neighbourhood of $r=r_{0}$. In principle, therefore, $U_{s}$ may still reach zero at a finite radius $r^{*}<r_{0}$, so long as the cut-off of $C_{s}$ occurs in $r^{*}<r<r_{0}$.

\subsection{The selected solution}

Finally we examine the possible asymptotic behaviour of solutions wherein $C_{s}$ stays bounded as $r \rightarrow r_{0}$. With the velocity field driven by a sink flow at infinity, we expect a Jeffery-Hamel-type flow as in Appendix C. However, any such flow inevitably has $\partial u / \partial z=O\left(1 /\left(r_{0}-r\right)^{2}\right)$ on $z=0$ which gives rise to the exponential growth (4.3) in $C_{s}$. The only way to avoid this is to choose the leading-order solution for $u$ such that 
$\partial u / \partial z \sim 0$ on $z=0$, that is

$$
U_{s} \sim u \sim \frac{\epsilon r_{0}}{\pi\left(r_{0}-r\right)} \text { as } r \rightarrow r_{0} .
$$

With $U_{s} \rightarrow \infty$ as $r \rightarrow r_{0}$, conservation of surfactant implies that $C_{s}$ must go to zero (order-of-magnitude arguments indicate that replenishment is subdominant). The lowest-order problem for $C$ is therefore

$$
\begin{gathered}
\frac{\epsilon r_{0}}{\pi}\left(\frac{1}{r_{0}-r} \frac{\partial C}{\partial r}-\frac{z}{\left(r_{0}-r\right)^{2}} \frac{\partial C}{\partial z}\right) \sim \frac{\partial^{2} C}{\partial z^{2}}, \\
C \rightarrow 1 \text { as } z \rightarrow \infty \\
C=0 \text { on } z=0,
\end{gathered}
$$

whose solution, in the limit $r \rightarrow r_{0}$, approaches the similarity solution

$$
C \sim \operatorname{erf}\left(\frac{z}{2\left(r_{0}-r\right)} \sqrt{\frac{\epsilon r_{0}}{\pi \log \left(k /\left(r_{0}-r\right)\right)}}\right)
$$

where the constant $k$ is arbitrary. It follows that

$$
\frac{\partial C}{\partial z} \sim \frac{1}{\pi\left(r_{0}-r\right)} \sqrt{\frac{\epsilon r_{0}}{\log \left(k /\left(r_{0}-r\right)\right)}} \text { on } z=0,
$$

and conservation of surfactant (2.15) then implies that

$$
C_{s} \sim 2 \beta\left(r_{0}-r\right) \sqrt{\frac{\log \left(k /\left(r_{0}-r\right)\right)}{\epsilon r_{0}}} .
$$

We test these asymptotic estimates in figure 12 . As noted in $\S 4.2$, the smallness of $\beta$ tends to obscure the local behaviour as $C_{s} \rightarrow 0$, so we artificially increase $\beta$ to 0.5 . We choose the value of $C_{0}$ for which (as closely as possible) $C_{s}$ is bounded as $r \rightarrow r_{0}$. The resulting behaviours of $U_{s}$ and $C_{s}$ are shown in the left-hand graph in figure 12. The right-hand graph shows plots of $\log \left(U_{s}\right)$ and $\log \left(C_{s}\right)$ versus $\log \left(r_{0}-r\right)$, along with our predictions

$$
\begin{aligned}
& \log \left(U_{s}\right) \sim-\log \left(r_{0}-r\right)+\log \left(\frac{\epsilon r_{0}}{\pi}\right) \\
& \log \left(C_{s}\right) \sim \log \left(r_{0}-r\right)+\frac{1}{2} \log \left(-\log \left(r_{0}-r\right)\right)+\log \left(\frac{2 \beta}{\sqrt{\epsilon r_{0}}}\right) .
\end{aligned}
$$



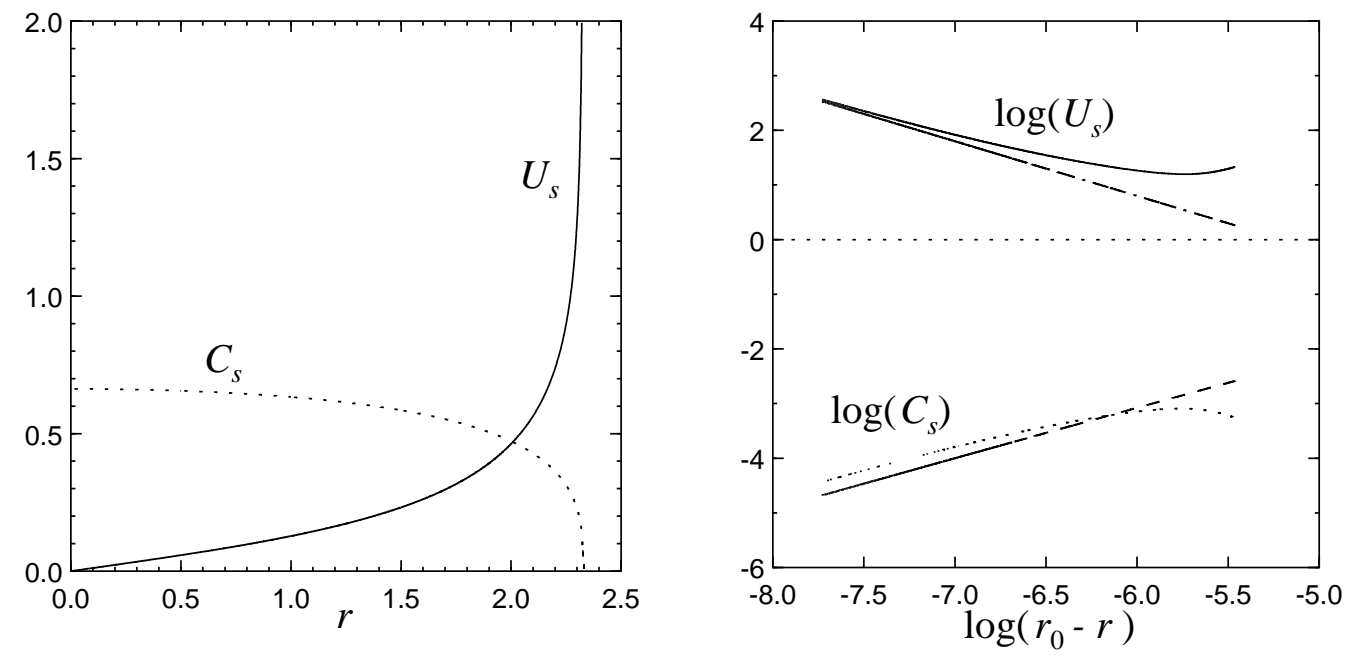

FIGURE 12. The left-hand graph shows the surface velocity $U_{s}$ and subsurface concentration $C_{s}$ versus $r$ for the parameter values $C_{b}=0.58 \mathrm{~mol} \mathrm{~m}^{-3}, a=0.04 \mathrm{~m}, \beta=0.5$ and $C_{0} \approx 0.663$. The right-hand graph shows the corresponding log-log plot; the dashed lines are the asymptotic predictions $(4.11,4.12)$.

The agreement appears to be good. Note that, while the error in our estimate (4.11) for $U_{s}$ is $O\left(r_{0}-r\right)$, that in $(4.12)$ is $O\left(1 / \log \left(r_{0}-r\right)\right)$ (associated with the free parameter $k)$, which is consistent with the discrepancy in figure 12 .

\section{Conclusions}

\subsection{Summary}

In $\S 2$, we presented a system of equations and boundary conditions governing the liquid velocity and surfactant concentration in the OFC. A detailed derivation of this model may be found in Breward et al. (2001), where it is shown that the equations do not specify a unique local behaviour near the axis of the cylinder: instead there is a one-parameter family of admissible solutions. Thus they were unable to predict directly how the surface concentration at the centre of the cylinder depends on the bulk concentration in solution, although this is one of the principal experimental results. 
Breward et al. (2001) suggested that a unique solution might be chosen by requiring the surfactant concentration to be finite as the edge of the cylinder is approached and nonzero everywhere else on the surface. To ascertain whether this selection mechanism applies to the OFC, we solved the model numerically in $\S 3$. The numerical solutions indicate that the general behaviour falls into one of three possible categories.

(a) A singularity forms a finite distance along the interface, where the surface concentration of surfactant goes to zero while the surface velocity approaches infinity.

(b) The surfactant concentration tends to infinity as the edge of the cylinder is approached, giving rise to a stagnation point a small distance upstream.

(c) These two generic solutions are separated by a distinguished case in which the surface concentration is zero at the edge of the cylinder.

We confirmed in $\S 4$ that each represents an admissible asymptotic solution of the governing equations, rather than a numerical artefact. The proposed selection mechanism is to insist on the third of these options, and this allows us to determine numerically a unique solution for any given experimental conditions.

\subsection{Comparison with experiment}

We are now in a position to compare the predictions of our model with experimental data. As a diagnostic we plot the (dimensional) surface concentration evaluated at the centre of the cylinder, $\Gamma(0)$, as a function of the bulk concentration $C_{b}$ in solution. The experimental results are shown as circles in figure 13 for a solution of CTAB in an overflowing cylinder of radius $a=4 \mathrm{~cm}$ with pump flux $Q_{p}=16 \mathrm{~cm}^{3} \mathrm{~s}^{-1}$ - see Bain et al. (2000) for more details about the experiments. It is thought that these values should be virtually independent of both $a$ and $Q_{p}$.

To compare with these data, we first have to evaluate the three parameters $\beta, r_{0}$ and $\epsilon$, all of which depend on the bulk concentration $C_{b}$. For each value of $C_{b}$, we pick $C_{0}$ 
$10^{6} \Gamma(0)$

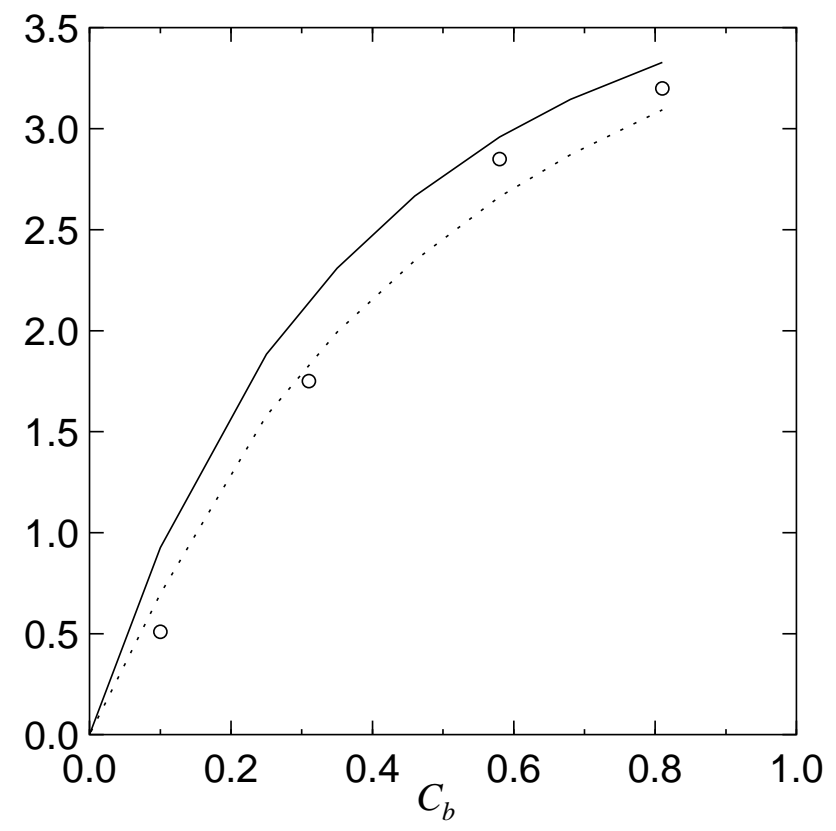

FiguRE 13. Dimensional surface concentration $\Gamma\left(\mathrm{mol} \mathrm{m}^{-2}\right)$, versus dimensional bulk concentration $C_{b}\left(\mathrm{~mol} \mathrm{~m}^{-3}\right)$. The circles show experimental values for a cylinder of radius $a=40 \mathrm{~mm}$ and pump flux $Q_{p}=16 \mathrm{~cm}^{3} \mathrm{~s}^{-1}$. The solid line shows the corresponding numerical solution. The dotted line shows the numerical solution for $a=25 \mathrm{~mm}, Q_{p}=8 \mathrm{~cm}^{3} \mathrm{~s}^{-1}$.

and then march downstream until either $C_{s}$ or $U_{s}$ reaches zero. We then vary $C_{0}$ in an attempt to locate the special solution that divides these two cases, and thus determine the axial surface concentration corresponding to each value of $C_{b}$. The relationship depends on both $a$ and the pump flux $Q_{p}$; we plot it for typical values thereof in figure 13 using a solid line $\left(a=4 \mathrm{~cm}, Q_{p}=16 \mathrm{~cm}^{3} \mathrm{~s}^{-1}\right)$ and a dotted line $\left(a=2.5 \mathrm{~cm}, Q_{p}=8 \mathrm{~cm}^{3} \mathrm{~s}^{-1}\right)$.

\subsection{Discussion}

Given the inaccuracy with which many of the physical parameters (e.g. $\left.D, \Gamma_{\text {sat }}, k\right)$ are known, there is remarkably good agreement between the experimental results and the numerical predictions, in terms of both order-of-magnitude and trend. Our model does predict a weak but systematic dependence on the cylinder radius which, according to 
Manning-Benson (1998), should not be there. This might be because our admittedly speculative selection criterion, namely insisting that $C_{s}$ be bounded as $r \rightarrow r_{0}$, is simply wrong. We have shown, however, that this criterion amounts to rejecting solutions in which either the surface concentration reaches zero at a finite distance along the free surface or an isolated stagnation point is formed inside the rim of the cylinder. To our knowledge, neither of these eventualities has been observed in experiments, and intuitively it seems unlikely that either could be physical. We therefore suspect that our selection mechanism is correct for our model equations, and that any disagreement between our predictions and experiment reflects shortcomings of the model. Our condition of zero concentration at the rim arises naturally from the mathematics, and leads to an experimentally sensible solution. It is not, though, at all obvious physically how the fluid manages to satisfy this downstream boundary condition on a parabolic problem. This puzzling feature clearly warrants further modelling and experimental investigation.

There are certainly many potentially important physical effects that we plan to consider in future refinements of the model. For example, many experiments have been carried out at sufficiently high bulk concentration that surfactant molecules in solution associate to form micelles. Incorporation of micellar effects in our equations is important both to interpret the results of such experiments and also because solutions above the critical micelle concentration are frequently used in practice.

It is also a priority to model the influence of electrostatic effects, since many practicallyimportant surfactants are ionic in character. This means that molecules adsorbed in the interface set up an electrical double layer that tends to inhibit the transport of further molecules from the bulk. Anomalous experimental results thought to be caused by ionic effects have already been observed in the OFC, especially at low bulk concentration (Bain et al. 2000). Another development of current experimental interest is the addition 
of further ionic species (e.g. salt) to the surfactant solution, the aim being to screen the effects of the double layer at the interface.

Other physical processes we have thus far neglected include surface diffusion and the possibility of the surface and bulk concentrations being out of thermodynamic equilibrium. Inclusion of such effects may regularise the singularities we have discovered. For example, the generation of singularities in the bulk concentration may be limited by the formation of micelles, while surface diffusion would smooth the behaviour of the surface concentration near the point where it vanishes. Preliminary investigations indicate, however, that none is able to resolve the indeterminacy in the solution near $r=0$; if anything they may make the situation worse. Some non-local criterion is therefore still required to select a unique solution.

The most suspect assumption made in our model is the zero-Froude-number limit, in which the free surface is perfectly flat and there is a line sink around the edge of the cylinder. Our defence for this approach is the reported lack of dependence of the results on the details of the flow over the rim. Nevertheless, the limit is certainly invalid near the rim and we suspect that this simplification, if anything, is responsible for systematic deviations between model and experimental data. In practice, the liquid flows over the edge at a nonzero (but small) height with a finite (but large) velocity. Presumably, under the action of the resultant high local strain rate, the concentration drops to a small (but nonzero) value. Our solution procedure, in which the concentration is set to zero at the rim, may therefore be regarded as the leading-order matching condition, for a singular perturbation in which the Froude number is the small parameter. For nonzero Froude number, the liquid-air interface is a free boundary and the problem is consequently very much harder to solve.

Finally, we reiterate the point that the singularities we have observed may arise in 
general surfactant flows. The first, due to the interaction between Marangoni acceleration of the interface and the consequent convection of surfactant, results in the surface concentration reaching zero a finite distance from the axis of the cylinder. The second singularity is caused by a strong gradient in the bulk flow, balanced by an exponentially large concentration gradient that gives rise to a stagnation point on the interface. The interesting integro-differential equation (C 21) holds the key to understanding whether or not the stagnation point forms. As argued in $\S 4.3$, it appears to be unnecessary for the subsurface velocity to be singular: a sufficiently rapid acceleration should suffice.

The authors would like to acknowledge the invaluable assistance of Dr C. D. Bain, Prof. R. C. Darton and Dr J. R. Ockendon in the preparation of this manuscript. We have also enjoyed many useful discussions with Prof. J. F. Harper, Dr S. D. Howison and Prof. O. E. Jensen.

\section{Appendix A. Approximation for the function $F(\rho)$}

To avoid having to evaluate the integral in (2.10) repeatedly, we employ an approximation that is obtained as follows. We expand (2.10) first as $\rho \rightarrow 0$ up to $O\left(\rho^{7}\right)$, then as $\rho \rightarrow 1$, keeping terms up to order $(1-\rho)^{3} \log (1-\rho)$. These two expressions are then combined to yield a composite expansion with the correct asymptotic behaviour near $\rho=0$ and $\rho=1$, namely

$$
\begin{aligned}
F(\rho) \approx & \frac{2 \rho}{\pi\left(1-\rho^{2}\right)} \\
& +\frac{3}{16 \pi}\{(1-\rho)(3-\rho) \log (1-\rho)-(1+\rho)(3+\rho) \log (1+\rho)\} \\
& +\left(b_{1}-\frac{7}{8 \pi}\right) \rho+\left(b_{3}-\frac{2}{\pi}\right) \rho^{3}+\left(b_{5}-\frac{81}{40 \pi}\right) \rho^{5} \\
& +\left(\frac{196}{5 \pi}+\frac{255}{16 \pi} \log 2-\frac{5 K}{2 \pi}+\frac{15 \varsigma}{16 \pi}-10 b_{1}-6 b_{3}-3 b_{5}\right) \rho^{7} \\
& +\left(-\frac{4361}{80 \pi}-\frac{387}{16 \pi} \log 2+\frac{9 K}{2 \pi}-\frac{27 \varsigma}{16 \pi}+15 b_{1}+8 b_{3}+3 b_{5}\right) \rho^{9}
\end{aligned}
$$




$$
+\left(\frac{1697}{80 \pi}+\frac{39}{4 \pi} \log 2-\frac{2 K}{\pi}+\frac{3 \varsigma}{4 \pi}-6 b_{1}-3 b_{3}-b_{5}\right) \rho^{11},
$$

where $\varsigma$ is Euler's constant,

$$
\begin{aligned}
b_{1} & =\frac{1}{2 \pi} \int_{0}^{\infty} \frac{k}{\mathrm{I}_{1}(k)} \mathrm{d} k \approx 0.88789 \\
b_{3} & =\frac{1}{16 \pi} \int_{0}^{\infty} \frac{k^{3}}{\mathrm{I}_{1}(k)} \mathrm{d} k \approx 0.67199 \\
b_{5} & =\frac{1}{384 \pi} \int_{0}^{\infty} \frac{k^{5}}{\mathrm{I}_{1}(k)} \mathrm{d} k \approx 0.64790 \\
K & =\int_{0}^{\infty}\left(\frac{k \mathrm{I}_{0}(k)}{\mathrm{I}_{1}(k)}-k-\frac{1}{2}-\frac{3}{8(1+k)}\right) \mathrm{d} k \approx 1.64464 .
\end{aligned}
$$

The relative error between (A 1) and (2.10) is everywhere less than $0.04 \%$.

\section{Appendix B. Asymptotic analysis as $C_{s} \rightarrow 0$}

We look for solutions in which $C_{s}$ reaches zero at $r=r^{*}$, as shown in figure 3 , while $U_{s}$ is large. A meaningful balance in $(2.4-2.16)$ consistent with the anticipated behaviour is obtained by rescaling as follows:

$$
\begin{gathered}
r=r^{*}-\delta \xi, \quad u=\delta^{-1 / 5} \tilde{u}, \quad U_{s}=\delta^{-1 / 5} \tilde{U}_{s} \\
z=\delta^{3 / 5} \zeta, \quad w=\delta^{-3 / 5} \tilde{w}, \quad C=\delta^{1 / 5} \tilde{C}, \quad C_{s}=\delta^{1 / 5} \tilde{C}_{s}
\end{gathered}
$$

where $\delta \ll 1$. We assume that the singularity develops a finite distance from the sink at $r=r_{0}$; we therefore need not worry about $F$ tending to infinity and may safely take $\epsilon \rightarrow 0$ for the present.

We rescale (2.4-2.16) using (B 1), set $\epsilon=0$ and throw away all terms of order $\delta$ or smaller. To lowest order, $(2.4,2.5)$ simplify to the two-dimensional boundary-layer equations:

$$
\begin{aligned}
-\tilde{u} \frac{\partial \tilde{u}}{\partial \xi}+\tilde{w} \frac{\partial \tilde{u}}{\partial \zeta} & =\frac{\partial^{2} \tilde{u}}{\partial \zeta^{2}}, \\
-\frac{\partial \tilde{u}}{\partial \xi}+\frac{\partial \tilde{w}}{\partial \zeta} & =0
\end{aligned}
$$


with boundary conditions

$$
\begin{gathered}
\tilde{u}=\tilde{U}_{s}(\xi) \text { on } \zeta=0, \\
\tilde{w}=0 \text { on } \zeta=0, \\
\tilde{u} \rightarrow 0 \text { as } \zeta \rightarrow \infty .
\end{gathered}
$$

The advection-diffusion equation for $C$ becomes

$$
-\tilde{U}_{s} \frac{\partial \tilde{C}}{\partial \xi}+\frac{\mathrm{d} \tilde{U}_{s}}{\mathrm{~d} \xi} \zeta \frac{\partial \tilde{C}}{\partial \zeta}=\frac{\partial^{2} \tilde{C}}{\partial \zeta^{2}}
$$

with boundary conditions

$$
\begin{array}{r}
\tilde{C}=\tilde{C}_{s}(\xi) \text { on } \zeta=0, \\
\delta^{3 / 5} \frac{\partial \tilde{C}}{\partial \zeta}=-\frac{\mathrm{d}}{\mathrm{d} \xi}\left(\frac{\tilde{U}_{s} \tilde{C}_{s}}{\beta+\delta^{1 / 5} \tilde{C}_{s}}\right) \text { on } \zeta=0, \\
\frac{\partial \tilde{u}}{\partial \zeta}=-\frac{1}{\beta+\delta^{1 / 5} \tilde{C}_{s}} \frac{\mathrm{d} \tilde{C}_{s}}{\mathrm{~d} \xi} \text { on } \zeta=0 .
\end{array}
$$

The rescaling of $C$ means that the boundary condition (2.13) at infinity cannot be imposed directly, as we will see below.

Now the behaviour near the singularity is obtained by seeking asymptotic expansions of the form $\tilde{u} \sim \tilde{u}_{0}+\delta^{1 / 5} \tilde{u}_{1}+\ldots$ and so forth. At leading order, the surfactant conservation boundary condition (B 9) is dominated by convection, with the implication that

$$
\tilde{U}_{s 0} \tilde{C}_{s 0}=Q
$$

for some positive constant $Q$ representing the flux of surfactant along the interface. Thus $\tilde{C}_{s 0}$ may be eliminated from (B 10) to give

$$
\frac{\partial \tilde{u}_{0}}{\partial \zeta}=\frac{Q}{\beta \tilde{u}_{0}^{2}} \frac{\partial \tilde{u}_{0}}{\partial \xi} \text { on } \zeta=0
$$

Equations (B 10) and (B 11) indicate the mechanism for the formation of a singularity. If diffusion is not strong enough to replenish the interface, then an increase in the surface velocity leads to a reduction in the surface concentration. The resulting Marangoni stress 
acts to accelerate the surface still further, resulting in a feedback loop. The boundary condition (B 12), along with (B 2-B 6), provides a closed system for the leading-order velocity, which admits a similarity solution of the form

$$
\begin{aligned}
\tilde{u}_{0} & =\phi^{\prime}(\eta), \quad \eta=\frac{\zeta}{\xi^{3 / 5}} \\
\tilde{w}_{0} & =\xi^{-3 / 5}\left(\frac{2}{5} \phi(\eta)-\frac{3}{5} \eta \phi^{\prime}(\eta)\right),
\end{aligned}
$$

where $\phi$ satisfies

$$
\begin{gathered}
\phi^{\prime \prime \prime}-\frac{2}{5} \phi \phi^{\prime \prime}-\frac{1}{5}\left(\phi^{\prime}\right)^{2}=0, \\
\phi(0)=0, \quad \phi^{\prime}(\infty)=0, \quad \phi^{\prime}(0) \phi^{\prime \prime}(0)=-\frac{Q}{5 \beta} .
\end{gathered}
$$

If $Q$ and $\beta$ are given, then (B 15) has a unique solution with asymptotic behaviour

$$
\phi \sim \alpha\left(\eta-\eta_{0}\right)^{2 / 3} \text { as } \eta \rightarrow \infty
$$

for some constants $\eta_{0}$ and $\alpha>0$. Therefore, if we define

$$
\phi=\alpha^{3 / 5} \Phi, \quad \eta=\eta_{0}+\alpha^{-3 / 5} Y
$$

then $\Phi(Y)$ satisfies the canonical problem

$$
\begin{gathered}
\Phi^{\prime \prime \prime}-\frac{2}{5} \Phi \Phi^{\prime \prime}-\frac{1}{5}\left(\Phi^{\prime}\right)^{2}=0, \\
\Phi \sim Y^{2 / 3}+\frac{2}{3 Y}-\frac{10}{7 Y^{8 / 3}}+\frac{1670}{189 Y^{13 / 3}}-\frac{2044415}{22491 Y^{6}}+\ldots \quad \text { as } \quad Y \rightarrow \infty .
\end{gathered}
$$

We solve (B 18) numerically, shooting from $Y \rightarrow \infty$, read off the value of $Y$ at which $\Phi=0\left(Y_{0} \approx-1.29059\right)$, and then obtain $\Phi^{\prime}\left(Y_{0}\right) \approx 0.71783$ and $-\Phi^{\prime \prime}\left(Y_{0}\right) \approx 0.16424$. We recover the value of $\alpha$ from (B 15)

$$
\alpha=\left(\frac{Q}{-5 \beta \Phi^{\prime}\left(Y_{0}\right) \Phi^{\prime \prime}\left(Y_{0}\right)}\right)^{1 / 3} \approx 1.1926\left(\frac{Q}{\beta}\right)^{1 / 3}
$$

By substituting into our similarity solution for $u$, we therefore obtain the leading-order surface velocity in the form

$$
\tilde{U}_{s 0}=A\left(\frac{Q}{\beta}\right)^{2 / 5} \xi^{-1 / 5}
$$


where

$$
A=\left(\frac{\Phi^{\prime}\left(Y_{0}\right)^{3}}{25 \Phi^{\prime \prime}\left(Y_{0}\right)^{2}}\right)^{1 / 3} \approx 0.8868
$$

From (B 11) we obtain the corresponding subsurface concentration,

$$
\tilde{C}_{s 0}=\frac{\beta^{2 / 5} Q^{3 / 5}}{A} \xi^{1 / 5}
$$

The leading-order concentration therefore satisfies the problem

$$
\begin{gathered}
\frac{\partial^{2} \tilde{C}_{0}}{\partial \zeta^{2}}=-A\left(\frac{Q}{\beta}\right)\left[\xi^{-1 / 5} \frac{\partial \tilde{C}_{0}}{\partial \xi}+\frac{\zeta}{5 \xi^{6 / 5}} \frac{\partial \tilde{C}_{0}}{\partial \zeta}\right], \\
\tilde{C}_{0}=\frac{\beta^{2 / 5} Q^{3 / 5}}{A} \xi^{1 / 5} \quad \text { on } \quad \zeta=0
\end{gathered}
$$

which suggests the similarity solution

$$
\tilde{C}_{0}=\xi^{1 / 5} \psi(\eta)
$$

It transpires that there is just one solution for $\psi$ that does not grow exponentially as $\eta \rightarrow \infty$, namely

$$
\psi(\eta)=\frac{5^{1 / 4} \gamma(1 / 4)}{A^{5 / 4} \sqrt{\pi}} \sqrt{\frac{\beta Q}{\eta}} \exp \left[\frac{A}{10}\left(\frac{Q}{\beta}\right)^{2 / 5} \eta^{2}\right] \mathcal{W}_{\frac{1}{2}, \frac{1}{4}}\left[\frac{A}{5}\left(\frac{Q}{\beta}\right)^{2 / 5} \eta^{2}\right],
$$

where $\gamma$ is the Gamma function and $\mathcal{W}$ is Whittaker's function (Gradshteyn \& Ryzhik 1994, page 1086), from which we deduce the behaviour

$$
\psi \sim \frac{\gamma(1 / 4)}{5^{1 / 4} A^{3 / 4} \sqrt{\pi}} Q^{7 / 10} \beta^{3 / 10} \sqrt{\eta} \text { as } \eta \rightarrow \infty .
$$

Clearly the original condition on $C$ at infinity cannot be applied directly. Instead there is an intermediate layer, where $C$ is $O(1)$. Although the solution in this outer region cannot be found locally, it is reassuring to confirm that a consistent asymptotic structure emerges from the rescaling

$$
Z=\delta^{2 / 5} \zeta=\delta^{-1 / 5} z
$$

resulting in

$$
-\tilde{U}_{s} \frac{\partial C}{\partial \xi}+\frac{\mathrm{d} \tilde{U}_{s}}{\mathrm{~d} \xi} Z \frac{\partial C}{\partial Z}=\delta^{4 / 5} \frac{\partial^{2} C}{\partial Z^{2}}
$$




$$
C \rightarrow 1 \text { as } Z \rightarrow \infty \text {. }
$$

Now, with $\tilde{U}_{s 0}$ given by (B 20), the general leading-order solution of (B 28) takes the form

$$
C_{0}=h\left(\frac{Z}{\xi^{1 / 5}}\right)
$$

The function of integration $h$ can only be determined by matching with the full solution upstream of the singularity. Nevertheless, it is clear that a solution of this form can be made to satisfy the condition at infinity and to match with the inner solution so long as $h$ satisfies

$$
\begin{array}{r}
h(\tau) \rightarrow 1 \text { as } \tau \rightarrow \infty, \\
h(\tau) \sim \frac{\gamma(1 / 4)}{5^{1 / 4} A^{3 / 4} \sqrt{\pi}} Q^{7 / 10} \beta^{3 / 10} \sqrt{\tau} \text { as } \tau \rightarrow 0 .
\end{array}
$$

\section{Appendix C. Asymptotic analysis of the sink}

As $r$ approaches $r_{0}$, the local behaviour of the flow is given by the two-dimensional boundary-layer equations driven by a sink at infinity:

$$
\begin{aligned}
u \frac{\partial u}{\partial r}+w \frac{\partial u}{\partial z}-\frac{\partial^{2} u}{\partial z^{2}} & \sim \frac{\epsilon^{2} r_{0}^{2}}{\pi^{2}\left(r_{0}-r\right)^{3}}, \\
\frac{\partial u}{\partial r}+\frac{\partial w}{\partial z} & \sim 0
\end{aligned}
$$

with

$$
\begin{array}{r}
u=U_{s}(r) \text { on } z=0, \\
w=0 \text { on } z=0, \\
u \sim \frac{\epsilon r_{0}}{\pi\left(r_{0}-r\right)} \text { as } z \rightarrow \infty .
\end{array}
$$

Our simulations indicate that, while the subsurface velocity approaches infinity, the surface velocity is unable to keep up and remains finite. Thus the dominant behaviour 
as $r \rightarrow r_{0}$ is a Jeffery-Hamel flow of the form

$$
u \sim \frac{\epsilon r_{0}}{\pi} \frac{G(\theta)}{r_{0}-r}, \quad w \sim-\frac{\epsilon r_{0}}{\pi} \frac{z G(\theta)}{\left(r_{0}-r\right)^{2}}, \quad \theta=\sqrt{\frac{\epsilon r_{0}}{2 \pi}}\left(\frac{z}{r_{0}-r}\right),
$$

where $G$ satisfies

$$
\begin{gathered}
G^{\prime \prime}=2\left(G^{2}-1\right), \\
G(0)=0, \quad G(\infty)=1,
\end{gathered}
$$

whose solution is

$$
G(\theta)=\frac{3 \tanh (\theta)[5 \tanh (\theta)+2 \sqrt{6}]}{[3+\sqrt{6} \tanh (\theta)]^{2}} .
$$

Consequently,

$$
\frac{\partial u}{\partial z}(r, 0) \sim\left(\frac{\epsilon r_{0}}{\pi}\right)^{3 / 2} \frac{G^{\prime}(0)}{\sqrt{2}\left(r_{0}-r\right)^{2}}=\left(\frac{\epsilon r_{0}}{\pi}\right)^{3 / 2} \frac{2}{\sqrt{3}\left(r_{0}-r\right)^{2}},
$$

and the tangential stress balance (2.16) may then be solved for the surface concentration:

$$
C_{s} \sim B \exp \left[\left(\frac{\epsilon r_{0}}{\pi}\right)^{3 / 2} \frac{2}{\sqrt{3}\left(r_{0}-r\right)}\right]
$$

for some constant $B$.

Thus $C_{s}$ approaches infinity exponentially quickly as the sink is approached. The mechanism is that the singular bulk flow applies a traction to the surface, and a surface concentration gradient must be set up to provide the balancing Marangoni stress. The surface velocity is then determined a posteriori from conservation of surfactant:

$$
\begin{gathered}
U_{s} \frac{\partial C}{\partial r}+\frac{\mathrm{d} U_{s}}{\mathrm{~d} r} z \frac{\partial C}{\partial z} \sim \frac{\partial^{2} C}{\partial z^{2}}, \\
C=C_{s}(r) \text { on } z=0, \\
\frac{\partial C}{\partial z} \sim \frac{\mathrm{d}}{\mathrm{d} r}\left(\frac{U_{s} C_{s}}{\beta+C_{s}}\right) \sim \frac{\mathrm{d} U_{s}}{\mathrm{~d} r} \text { on } z=0, \\
C \rightarrow 1 \text { as } z \rightarrow \infty .
\end{gathered}
$$

With $C_{s}$ given asymptotically by (C 10), (C 11, C 12, C 14) would appear to be a wellposed problem for $C$, except that the surface velocity $U_{s}$ is not known in advance: it must be solved for as part of the problem by using (C 13). 
Since the concentration at $z=0$ grows without bound while that at infinity is finite, we expect the gradient $\partial C / \partial z$ to become large and negative as the sink is approached. If so, then (C 13) implies that $\mathrm{d} U_{s} / \mathrm{d} r$ is likewise large and negative as $r \rightarrow r_{0}$. This may mean that $U_{s}$ approaches zero or a finite value with infinite slope as $r \rightarrow r_{0}$, or that $U_{s}$ becomes zero at a finite value of $r<r_{0}$. Further evidence as to which of these is correct is obtained as follows.

The transformation

$$
t=\int_{0}^{r} U_{s}(\rho) \mathrm{d} \rho, \quad y=z U_{s}(r)
$$

reduces the advection-diffusion equation $(\mathrm{C} 11)$ to the heat equation

$$
\frac{\partial C}{\partial t}=\frac{\partial^{2} C}{\partial y^{2}}
$$

while (C 14) implies that

$$
\begin{aligned}
& C \rightarrow 1 \text { as } y \rightarrow \infty, \\
& C=1 \text { at } t=0 .
\end{aligned}
$$

With $C=C_{s}$ on $y=0$, the solution may be written as

$$
C=1-\frac{y}{2 \sqrt{\pi}} \int_{0}^{t}\left[1-C_{s}(r(\tau))\right] \exp \left(-\frac{y^{2}}{4(t-\tau)}\right) \frac{\mathrm{d} \tau}{(t-\tau)^{3 / 2}},
$$

and hence

$$
\frac{\partial C}{\partial y}=\frac{1}{\sqrt{\pi}} \frac{\mathrm{d}}{\mathrm{d} t} \int_{0}^{t}\left[1-C_{s}(r(\tau))\right] \frac{\mathrm{d} \tau}{\sqrt{t-\tau}} \text { on } y=0
$$

By substituting this into $(\mathrm{C} 13)$ and using the fact that $\mathrm{d} r / \mathrm{d} t=U_{s}^{-1}$, we obtain a nonlinear Abel integro-differential equation for $r$ as a function of $t$ :

$$
\frac{\mathrm{d} r}{\mathrm{~d} t}=\sqrt{\pi}\left\{\int_{0}^{t}\left[1-C_{s}(r(\tau))\right] \frac{\mathrm{d} \tau}{\sqrt{t-\tau}}\right\}^{-1} .
$$

With $C_{s}$ assumed to be a known function of $r$ and $r(0)=0,(\mathrm{C} 21)$ may be solved as an initial-value problem for $r(t)$. 
We suppose that $C_{s}$ is bounded for $r<r_{0}$; it follows that the integral in (C21) is also bounded for all $r<r_{0}$ and therefore that $\mathrm{d} r / \mathrm{d} t$ cannot change sign without first becoming infinite (when the integral passes through zero). It is therefore impossible for $r$ to tend to a finite value as $t \rightarrow \infty$ and the only remaining possibilities are (i) $r=r_{0}$ at a finite value of $t$ or (ii) $\mathrm{d} r / \mathrm{d} t$ reaches infinity at a finite value of $t$ and $r<r_{0}$. The former of these would correspond to the surface velocity attaining a finite value (possibly zero) at the sink $r=r_{0}$, while the latter would imply that $U_{s}$ reaches zero at a finite value of $r<r_{0}$

Numerical experiments performed on (C 21) indicate that, although (i) may arise from less singular $C_{s}(r)$, the form given in (C 10) always leads to option (ii). Indeed, with $C_{s}$ given asymptotically by (C10), one can argue that it is impossible for $r$ to reach $r_{0}$ as follows. Suppose $r\left(t^{*}\right)=r_{0}$ for some finite value $t^{*}$. For the integral in (C 21) to be finite, $r$ must approach $r_{0}$ very slowly, with

$$
r_{0}-r \gtrsim \frac{\text { const }}{-\log \left(t^{*}-t\right)} \text { as } t \rightarrow t^{*}
$$

and, therefore,

$$
\frac{\mathrm{d} r}{\mathrm{~d} t} \gtrsim \frac{\text { const }}{\left(t^{*}-t\right) \log ^{2}\left(t^{*}-t\right)} \text { as } t \rightarrow t^{*}
$$

Using this, we deduce an estimate for the local behaviour of the integral in (C 21):

$$
\int_{t}^{t^{*}} \frac{C_{s}(r(\tau)) \mathrm{d} \tau}{\sqrt{t^{*}-\tau}} \lesssim \operatorname{const}\left(t^{*}-t\right) \log ^{2}\left(t^{*}-t\right) \text { as } t \rightarrow t^{*}
$$

which appears to be inconsistent with $C_{s} \rightarrow \infty$ as $t \rightarrow t^{*}$.

We claim, therefore, that $U_{s}$ reaches zero at a finite value of $r<r_{0}$, beyond which the solution cannot be continued. The behaviour of $U_{s}$ near the spontaneously-formed stagnation point as follows. Suppose that $U_{s}=0$ at $r=r^{*}=r\left(t^{*}\right)$ so that, from (C 21),

$$
\int_{0}^{t^{*}} \frac{C_{s}(r(\tau))}{\sqrt{t^{*}-\tau}} \mathrm{d} \tau=2 \sqrt{t^{*}}
$$


and that $r$ has the local behaviour

$$
r(t) \sim r^{*}-A\left(t^{*}-t\right)^{\alpha} \text { as } t \rightarrow t^{*}
$$

for some positive constant $A$ and $\alpha \in(0,1 / 2)$. The left- and right-hand sides of (C 21) may then be expanded locally to give

$$
A \alpha\left(t^{*}-t\right)^{\alpha-1} \sim \frac{\gamma(-\alpha)}{A C_{s}^{\prime}\left(r^{*}\right) \gamma(-\alpha-1 / 2)}\left(t^{*}-t\right)^{-\alpha-1 / 2}
$$

where $C_{s}^{\prime}\left(r^{*}\right)=\mathrm{d} C_{s} / \mathrm{d} r\left(r^{*}\right)$ and, therefore,

$$
\alpha=\frac{1}{4}, \quad A=\frac{\sqrt{6 \sqrt{2}} \gamma(3 / 4)}{\sqrt{\pi C_{s}^{\prime}\left(r^{*}\right)}} .
$$

The corresponding behaviour of $U_{s}(r)$ is (4.4).

It is worth pointing out that our prediction $(\mathrm{C} 10)$ of the behaviour of $C_{s}$ is obtained in the limit $r \rightarrow r_{0}$, even though we have argued that the solution never reaches $r_{0}$. Thus our estimates only apply if $\epsilon$ is small, so that $r^{*}$ is close to $r_{0}$.

\section{REFERENCES}

Adamson, A. W. 1982 Physical Chemistry of Surfaces, 4th edn. Wiley.

Atkins, P. W. 1992 The Elements of Physical Chemistry. Oxford.

Bain, C. D., Manning-Benson, S. \& Darton, R. C. 2000 Rates of mass transfer and adsorption of hexadecyltrimethylammonium bromide at an expanding air-water interface. $J$. Coll. Int. Sci. 229, 247-256.

Breward, C. J, W., Darton, R. C., Howell, P. D. \& Ockendon, J. R. 2001 The effect of surfactants on expanding free surfaces. Chem. Eng. Sci. 56(8), 2867-2878.

Breward, C. J. W. \& Howell, P. D. 2002 The drainage of a foam lamella. J. Fluid Mech. 458, 379-406.

Di Pietro, N. D. \& Cox, R. G. 1980 The containment of an oil slick by a boom placed across a uniform stream. J. Fluid Mech. 96, 613-640.

Di Pietro, N. D., Huh, C. \& Cox, R. G. 1978 The hydrodynamics of the spreading of one liquid on the surface of another. J. Fluid Mech. 84, 529-549. 
Gradshteyn, I. S. \& Ryzhik, I. M. 1994 Table of Integrals, Series, and Products, 5th edn. Academic.

Halpern, D. \& Grotberg, J. B. 1992 Dynamics and transport of a localised soluble surfactant on a thin film. J. Fluid Mech. 237, 1-11.

HARPER, J. F. 1992 The leading edge of an oil slick, soap film, or bubble stagnant cap in Stokes flow. J. Fluid Mech. 237, 23-32.

Harper, J. F. \& Dixon, J. N. 1974 The leading edge of a surface film on contaminated flowing water. Proc. Fifth Australasian Conf. Hydraulics Fluid Mech., Christchurch, NZ vol. 2, pp. 499-505.

Jensen, O. E. 1995 The spreading of insoluble surfactant at the free surface of a deep fluid layer. J. Fluid Mech. 293, 349-378.

Jensen, O. E. 1998 The stress singularity in surfactant-driven thin-film flows. Part 2. Inertial effects. J. Fluid Mech. 372, 301-322.

Jensen, O. E. \& Grotberg, J. B. 1993 The spreading of heat or soluble surfactant on a thin liquid film. Phys. Fluids A 5, 58-68.

Jensen, O. E. \& HALPern, D. 1998 The stress singularity in surfactant-driven thin-film flows. Part 1. Viscous effects. J. Fluid Mech. 372, 273-300.

Manning-Benson, S., 1998 The Dynamics of Surfactant Adsorption. DPhil thesis, University of Oxford.

Manning-Benson, S., Bain, C. D. \& Darton, R. C. 1997 a Measurement of dynamic interfacial properties in an overflowing cylinder by ellipsometry. J. Coll. Int. Sci. 189, 109-116.

Manning-Benson, S., Bain, C. D., Darton, R. C., Sharpe, D., Eastoe, J. \& REYNOLDS, P. $1997 b$ Invasive and non-invasive measurements of dynamic surface tensions. Langmuir 13, 5808-5810.

Manning-Benson, S., Parker, S. W. R., Bain, C. D. \& Penfold, J. 1998 Measurement of the dynamic surface excess in an overflowing cylinder by neutron reflection. Langmuir 14, 990-996. 